\title{
Morphological Structures and Drug Release Effect of Multiple Electrospun Nanofibre Membrane Systems Based on PLA, PCL, and PCL/Magnetic Nanoparticle Composites
}

\author{
Hazim J. Haroosh, ${ }^{1}$ Yu Dong $\mathbb{D},{ }^{1}$ Shaimaa Jasim, ${ }^{2}$ and Seeram Ramakrishna ${ }^{3}$ \\ ${ }^{1}$ School of Civil and Mechanical Engineering, Curtin University, Perth, WA 6845, Australia \\ ${ }^{2}$ Department Biomedical Science, Murdoch University, Perth, WA, Australia \\ ${ }^{3}$ Department of Mechanical Engineering, National University of Singapore, Singapore 117575, Singapore \\ Correspondence should be addressed to Yu Dong; y.dong@curtin.edu.au
}

Received 7 October 2021; Revised 24 December 2021; Accepted 27 January 2022; Published 16 February 2022

Academic Editor: Dong Kee Yi

Copyright (c) 2022 Hazim J. Haroosh et al. This is an open access article distributed under the Creative Commons Attribution License, which permits unrestricted use, distribution, and reproduction in any medium, provided the original work is properly cited.

\begin{abstract}
Biopolymers are good carrier materials in relation to efficient release sustainability for encapsulated drugs. In particular, electrospun polymer/composite fibre membranes can offer greater benefits owing to their competitive release features as well as large specific surface areas. In this study, multiple electrospun nanofibre membrane systems were utilised including different material systems such as poly(lactic acid) (PLA), poly( $\varepsilon$-caprolactone) (PCL), and PCL/magnetic nanoparticle (MP) composites loaded with tetracycline hydrochloride $(\mathrm{TCH})$ as a therapeutic compound for their potential use in drug delivery applications. Such electrospun nanofibres were investigated to understand how composite constituents could tailor surface morphology for drug release control and biodegradation effect of PCL electrospun nanofibers on a long term for different drug release systems. Fibre diameter appeared to be decreased considerably with the addition of TCH drug. It was also evident that average fibre diameter was reduced when embedding MPs owing to the enhancement of solution conductivity. The encapsulation of TCH drug was found to be effective, as evidenced by Fourier transform infrared (FTIR) spectra. Thermogravimetric analysis (TGA) data revealed no significant change in the thermal stability of PCL with the inclusion of TCH and MPs. However, the use of TCH to PLA delayed the thermal degradation. Glass transition temperature $\left(T_{g}\right)$ and melting temperature $\left(T_{m}\right)$ of PCL were decreased with the inclusion of MPs and TCH. The degree of crystallinity $\left(X_{c}\right)$ for PCL diminished when incorporated with MPs. Additional TCH to PLA, PCL, and PCL/MP nanocomposites resulted in a moderate decrease in $X_{c}$. TCH might be dispersed in an amorphous state within nanofibre membranes. Over the short-term periods, it was clearly seen that TCH release from PCL nanofibre membranes was higher as opposed to PLC/MP and PLA counterparts. On the contrary, such a drug release from PLC membranes became relatively slow owing to its high $X_{c}$. Further, the mass loss results were consistent with those obtained from in vitro drug release. Overall, TCH release kinetics of PCL/TCH nanofibre membranes were better estimated by Zeng model as opposed to PLA/TCH counterparts.
\end{abstract}

\section{Introduction}

Electrospinning is a material fabrication technique that produces functional and economical three-dimensional fibrous structures $[1,2]$. The electrospun fibres result in the formation of a fabric network in possession of high porosity, small pore size, and large surface area to volume ratio $[3,4]$. In recent years, this technique has been uti- lised for many biomedical applications particularly in controlling drug delivery systems $[5,6]$. Biopolymers have been frequently employed for multiple purposes in pharmaceutical applications such as tissue engineering and drug delivery. Several biodegradable polymers have been fabricated for drug delivery such as poly(lactic acid) (PLA) [7-9], poly( $\varepsilon$-caprolactone) (PCL) [10], poly(glycolic acid) (PGA), poly(lactide-co-glycolide) (PLGA) and 
polyurethane (PU), as well as natural proteins including collagen, gelatin, and elastin [1].

Poly(lactic acid) (PLA) is a biodegradable polyester derived from $\alpha$-hydroxy acids with good mechanical properties and several potential applications [11], as exemplified by a dialysis medium, a matrix for tissue engineering, biodegradable sutures, agricultural products, drug delivery systems, food packaging and consumer products [12]. PLA has remarkable properties like easy processability, biodegradability $[13,14]$, the ability to be dissolved in common solvents, good sustainability and reasonable optical properties [12]. On the flip side, PLA has typical brittleness and slow crystallisation [15], and its degradation can be harmful to local tissues [16] owing to its low $\mathrm{pH}$ level [17].

Poly( $\varepsilon$-caprolactone) (PCL) is a hydrophobic and semicrystalline polymer [5] mainly for the purpose of enhancing the elasticity of materials [18]. It is well-known for its acceptable drug permeability and good biocompatibility $[19,20]$. The typical material merit of PCL, which is known that degradation is unable to generate a local acidic environment along with moderately low cost, makes it a favourable candidate for biomedical applications [5, 21]. The Food and Drug Administration (FDA) approved the safe use of PCL for a variety of industrial applications such as medical devices, wound dressing, resorbable membranes, cosmetics, filtration membranes, packaging, and agricultural materials [22]. The degradation rate of PCL appears to be relatively slow when compared with PLA [23]. PCL has little harm to local tissues and overcomes the drawback of PLA to generate an acidic environment. Nonetheless, it is worth mentioning that slow degradation, poor porosity and semicrystalline nature of PCL inevitably hinder its wide use in the field of drug delivery. PCL possesses high crystallinity and thus easily restricts the mobility of drug molecules that would tend to stay on the fibre surfaces [6]. As such, PCL is generally mixed with additives or other biopolymers to overcome such a critical issue like electrospun composite nanofibres based on PCL/collagen, PCL/gelatin, and PCL/ multiwalled carbon nanotubes (MWCNTs) [24].

On the other hand, $\mathrm{Fe}_{3} \mathrm{O}_{4}$ has been widely investigated and used among magnetic nanoparticles for bioscience applications [25]. Such an inorganic material has low toxicity, super paramagnetic properties, good biocompatibility and better stability in atmosphere as opposed to metal nanoparticles. These magnetic nanoparticles are also potentially helpful in various fields such as data storage, sensors [26], purification separation, magnetic resonance imaging, enzyme immobilisation, immunoassay, drug delivery and hyperthermia treatment [27]. A simple method to create $\mathrm{Fe}_{3} \mathrm{O}_{4}$ nanoparticles for their encapsulation in electrospun polyvinyl pyrrolidone (PVP) nanofibres was developed in the previous work [25] in order to investigate their magnetic properties and structures. With increasing the repulsive forces in electrospinning, the electrostatically driven instability is enhanced, thus resulting in a more whipping phenomenon for the jet to reduce fibre diameter [28].

Tetracycline hydrochloride (TCH) is an antibiotic drug utilised for the curing and avoidance of bacterial infections in the burn, injury and surgery in addition to numerous wound healing areas [6]. The objective of this study is to evaluate the fabrication, structural and thermal properties and biodegradability of electrospun PLA-based or PCLbased fibre membranes, $\mathrm{TCH}$ drug release with respect to electrospun PLA/TCH, PCL/TCH and PCL/MP/TCH nanofibre composite membranes, as well as further fabrication of novel carrier structures in order to achieve efficient drug delivery. In addition, this study also attempts to tackle the problem regarding the slow TCH release from PCL on the long term as a result of slow nanofibre degradation and its high $X_{c}$ when compared to PLA where MPs are added to increase the surface area and porosity of electrospun nanofibres. Finally, mathematical modelling has also been employed for better understanding of drug release kinetics.

\section{Materials and Fabrication}

2.1. Materials. PLA 3051D with the molecular weight (MW) of $93,500 \mathrm{~g} / \mathrm{mol}$ was supplied by the NatureWorks, USA. PCL (MW $=80,000 \mathrm{~g} / \mathrm{mol})$, iron sulfate, iron nitrate, tetracycline hydrochloride (TCH) in a chemical formula of $\mathrm{C}_{22} \mathrm{H}_{24} \mathrm{~N}_{2} \mathrm{O}_{8} \cdot \mathrm{HCl}$ and $\mathrm{MW}=480.9 \mathrm{~g} / \mathrm{mol}$, phosphate buffer solution (PBS), chloroform, and methanol were purchased from Sigma-Aldrich Ltd, Australia, which were used without any purification. Iron sulfate and iron nitrate were employed to synthesise MPs while TCH and PBS acted as a model drug and a medium of drug release, respectively. The solvents selected in all cases were a mixture of chloroform and methanol processed at a constant volume ratio of $2: 1$.

2.2. Electrospinning. Electrospinning was carried out using both $8 \mathrm{wt} \% / \mathrm{v}$ PLA and PCL solutions. MPs were synthesised from iron sulfate $\left(\mathrm{FeSO}_{4}\right)$ and iron nitrate $\left(\mathrm{Fe}\left(\mathrm{NO}_{3}\right)_{3}\right)$ and were further added at $0.1 \mathrm{wt} \% / \mathrm{v}$ to the PCL solution, which underwent $2 \mathrm{~h}$ ultrasonication. $5 \mathrm{wt} \% / \mathrm{v}$ TCH solution was mixed with $0.1 \mathrm{wt} \% / \mathrm{v}$ MPs for $2 \mathrm{~h}$ in methanol by using a similar ultrasonication process and subsequently added to PLA, PCL and PCL/MP solutions. Prior to the electrospinning process, finally prepared solution was transferred to a $10 \mathrm{ml}$ syringe mounted onto a Fusion 100 syringe pump (Chemyx Inc. Stafford, TX, USA) with a $20 \mathrm{G}$ metallic needle (inner diameter: $0.584 \mathrm{~mm}$ ). The flow rate of polymer solution was set at $2 \mathrm{ml} / \mathrm{h}$, and the applied positive voltage was $25 \mathrm{kV}$. The electrospinning process was conducted at $24^{\circ} \mathrm{C}$. The resulting fibres were collected on a ground collector covered by a flat aluminium foil. The distance between the needle tip and the target was $13 \mathrm{~cm}$.

2.3. In Vitro Drug Release Study. The drug-loaded nanofibre membrane sample $\left(2 \times 2 \mathrm{~cm}^{2}\right)$ was incubated in a rotary shaker at $37^{\circ} \mathrm{C}$ in $20 \mathrm{ml} \mathrm{PBS}(\mathrm{pH}=7.4)$. After the required incubation time for drug release, the sample was transferred to $20 \mathrm{ml}$ fresh buffer solution, and the amount of released $\mathrm{TCH}$ in the buffer solution was determined. A standard release curve was obtained by measuring different concentrations of TCH/PBS with the aid of a UV-vis spectrometer at the wavelength of $360 \mathrm{~nm}$. The percentage of the released drug was calculated from the primary weight of TCH incorporated into electrospun nanofibre membranes. The 
cumulative amount of drug released from nanofibre membranes was calculated according to Equation (1) [6]:

$$
\text { Cumulative quantity of drug released }(\%)=\frac{M_{t}}{M_{\infty}} \times 100 \% \text {, }
$$

in which $M_{t}$ is the amount of drugs released up to time $t$, and $M_{\infty}$ is the initial amount of drug within electrospun nanofibres. Three release tests were conducted for each electrospun nanofibre membrane, and the average data were recorded accordingly.

2.4. Zeng Model. Zeng model has been used to interpret the drug release mechanism [29]. The equation for Zeng model is relatively complex, as shown in Equations (2) and (3) [29].

$$
\frac{M_{t}}{M_{\infty}}=\frac{K_{\text {off }}}{K_{\text {on }}+K_{\text {off }}}\left(1-e^{-K_{s} t}\right)+\frac{K_{\text {on }}}{K_{\text {on }}+K_{\text {off }}}\left(1-e^{-K_{\text {off }} t}\right),
$$

$$
\Delta G=-k_{B} T\left(\frac{K_{\text {on }}}{K_{\text {off }}}\right),
$$

where $K_{\text {on }}$ is the rate constant of association for nondispersed drug molecules in the system with the requirement of being disassociated from the carriers prior to drug release. On the other hand, $K_{\text {off }}$ is the rate constant of disassociation accordingly, and $K_{S}$ is a constant proportional to the surface-to-volume ratio of the carriers for enhancing the drug release. $\Delta G$ is the free energy difference between free and bound states. Whereas, $k_{B}$ is Boltzmann's constant for which the absolute temperature is assumed to be $300 \mathrm{~K}$.

2.5. In Vitro Biodegradation Study. With respect to in vitro degradation studies, nanofibre membranes with the fibre thicknesses ranging from 300 to $450 \mu \mathrm{m}$ were sliced into 2 $\times 2 \mathrm{~cm}$ testing samples. They were measured to determine the initial weight $(m)$ and then were placed into an incubated rotary shaker at the rotor speed of $100 \mathrm{rpm}$ during the biodegradation process at $37^{\circ} \mathrm{C}$ using $15 \mathrm{ml} P B S$ $(\mathrm{pH}=7.4)$ for the required incubation time.

Such nanofibre membranes were removed at each designated incubation period and washed with deionised water. These membranes were dried under vacuum at $37^{\circ} \mathrm{C}$ until they reached a constant weight $\left(m_{1}\right)$. Mass loss (\%) was determined using Equation (4) [6]:

$$
\text { Mass loss }(\%)=\left(\frac{m-m_{1}}{m}\right) \times 100 \% \text {. }
$$

\section{Characterisation Techniques}

Solution viscosity was evaluated with a Visco 88 portable viscometer from Malvern Instruments (UK). Nanofibre morphology was studied via an EVO 40XVP scanning electron microscope (SEM) (Germany) at an accelerating voltage of $5 \mathrm{kV}$. Before SEM observation, the samples were sputter-coated with platinum. Fibre diameter was calculated from SEM images by using an image analysis tool based on Zeiss smart SEM software. For each sample, the measurements were made from a minimum of 150 fibres from multiple scanned SEM images at a sampling rate of 15 fibres per image. On the other hand, Fourier transform infrared spectroscopy (FTIR) was performed in a Spectrum 100 FTIR Spectrometer (Perkin Elmer, Japan). The associated spectra were recorded in a wavelength range of $4000-550 \mathrm{~cm}^{-1}$ with $4 \mathrm{~cm}^{-1}$ resolution according to an attenuated total reflectance (ATR) technique. Moreover, thermogravimetric analysis (TGA) was carried out by using a SeikoSII Exstar 6000 (TG/DTA 6200) to detect thermal decomposition effect. About 6-10 mg TGA samples were heated from 40 to $900^{\circ} \mathrm{C}$ at a heating ramp rate of $10^{\circ} \mathrm{C} / \mathrm{min}$ in the nitrogen flow of $200 \mathrm{ml} / \mathrm{min}$. Thermal analysis was performed using a DSC6000 instrument (Perkin Elmer, USA) with a cryofill liquid nitrogen cooling system. Approximately $10 \mathrm{mg}$ of fibre membrane was cut and sealed in an aluminum pan. The thermal behaviour was analysed during the first heating scan in a temperature range from $-90^{\circ} \mathrm{C}$ to $200^{\circ} \mathrm{C}$ at the ramp rate of $10^{\circ} \mathrm{C} / \mathrm{min}$. X-ray diffraction (XRD) measurements of prepared samples were undertaken in a Bruker Discover 8 X-ray diffractometer (Bruker Corporation, Germany) operated at $40 \mathrm{kV}$ and $40 \mathrm{~mA}$ using $\mathrm{Cu}-\mathrm{K} \alpha$ radiation subjected to the monochromatisation with graphite sample monochromators in a $2 \theta$ range from $5^{\circ}$ to $40^{\circ}$ (scanning rate: $0.05^{\circ} / \mathrm{s}$ ). Finally, the amount of TCH present in the release buffer was determined by a JascoV-67 UV-vis spectrophotometer at the wavelength of $360 \mathrm{~nm}$.

\section{Results and Discussion}

4.1. Fibre Morphology. To investigate the effect of nanoparticles and drug on the morphology, PCL, PCL/MPs, PCL/ MPs/TCH, PLA and PLA/TCH nanofibres were fabricated. As illustrated in Figure 1, electrospun nanofibres produced from PCL and PLA have the average fibre diameters of 368 \pm 15 and $510 \pm 20 \mathrm{~nm}$, respectively. The addition of MPs to PCL enhances electrical conductivity, which is attributed to an increase in iron content. PCL/MP composite system appears to create fibrous structures with smaller fibre diameters of $316 \pm 20 \mathrm{~nm}$ as opposed to those of electrospun PCL fibres. When the solution electrical conductivity increases, more electric charges are carried by an electrospinning jet. As such, higher repulsive forces are imposed to the jet in the electrical field. On the other hand, by increasing the solution conductivity, bending instability can be increased during electrospinning. As a result, the jet path becomes much longer, and more stretching of solution droplets can be induced. Both higher repulsive forces and greater bending instability can make a significant contribution to electrospun fibres with corresponding lower fibre diameters [30]. Table 1 summarizes average nanofibre diameters for all fibre membranes prepared in this study.

On the other hand, it is manifested that fibre diameters were reduced significantly from $368 \pm 15$ to $279 \pm 15 \mathrm{~nm}$ for PCL, from $316 \pm 20$ to $225 \pm 15 \mathrm{~nm}$ for PCL/MPs and from $510 \pm 20$ to $397 \pm 20 \mathrm{~nm}$ for PLA accordingly when loaded with TCH drug. This decrease in nanofibre diameter 


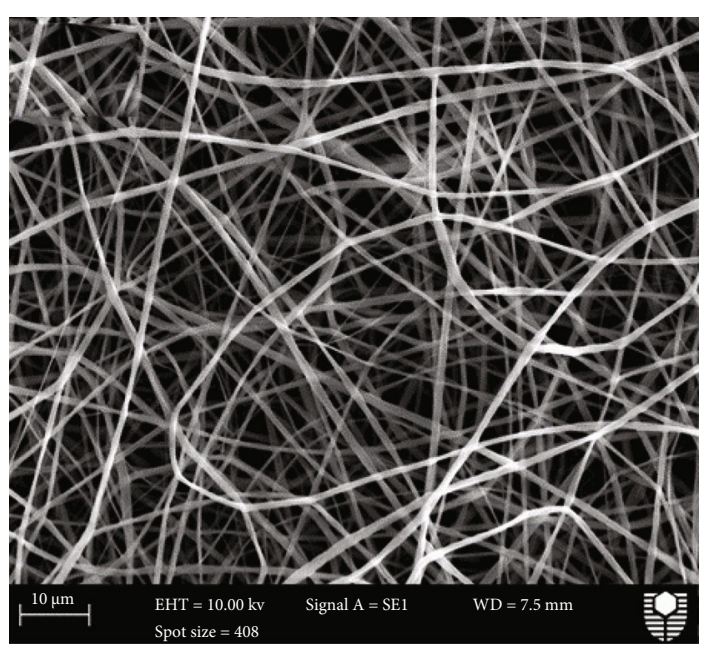

(a)

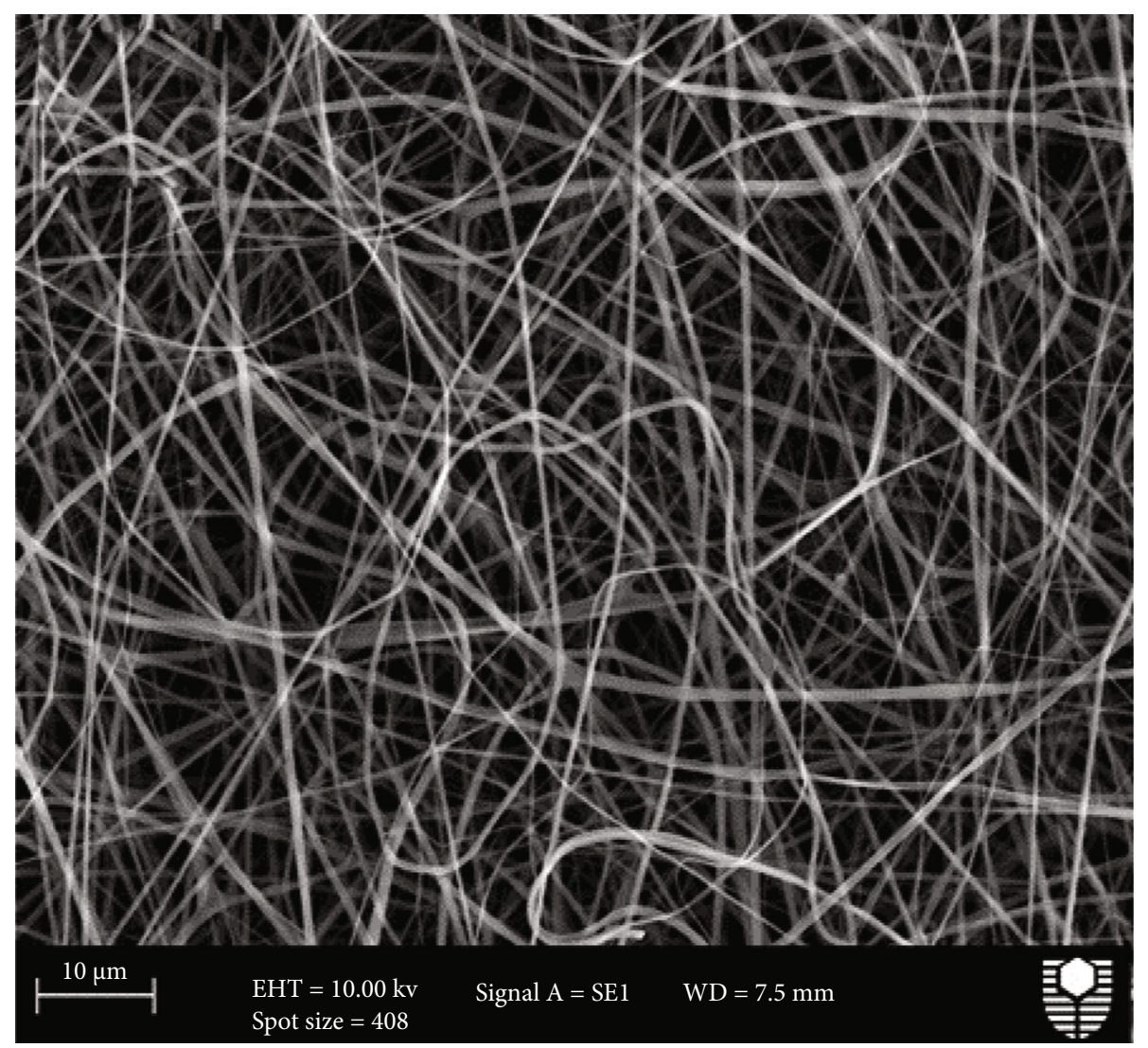

(b)

Figure 1: Continued. 


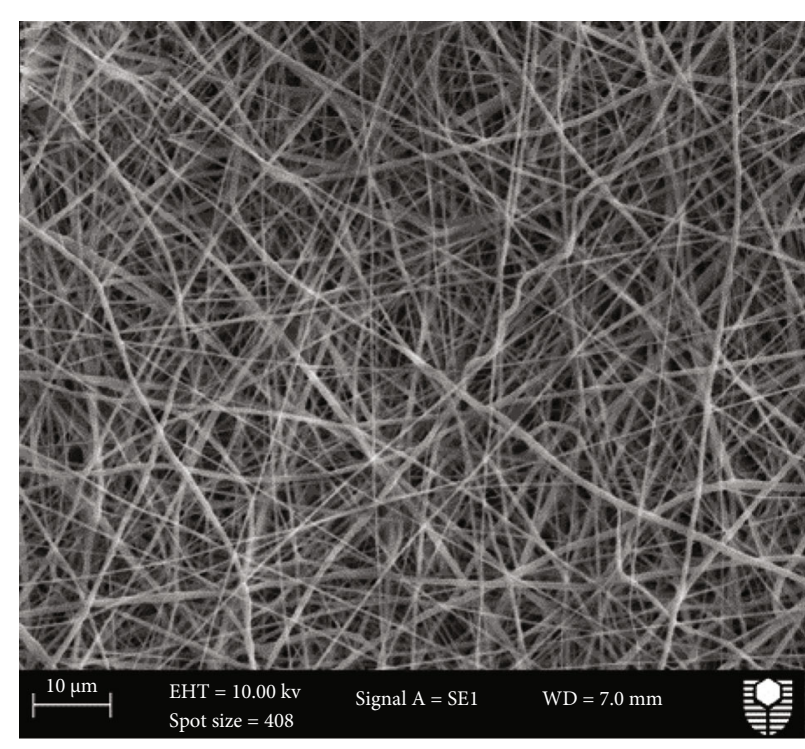

(c)

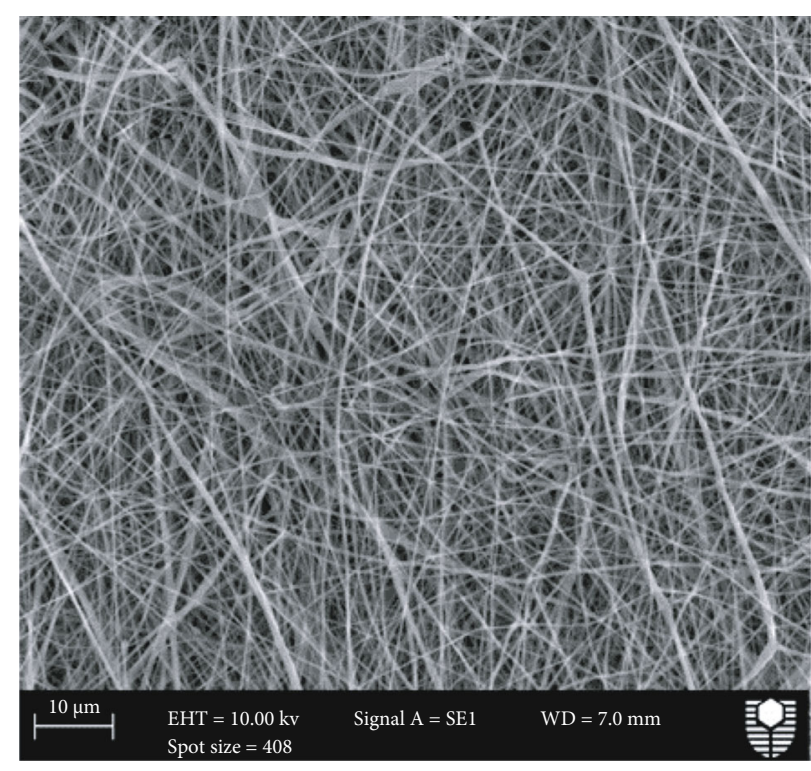

(d)

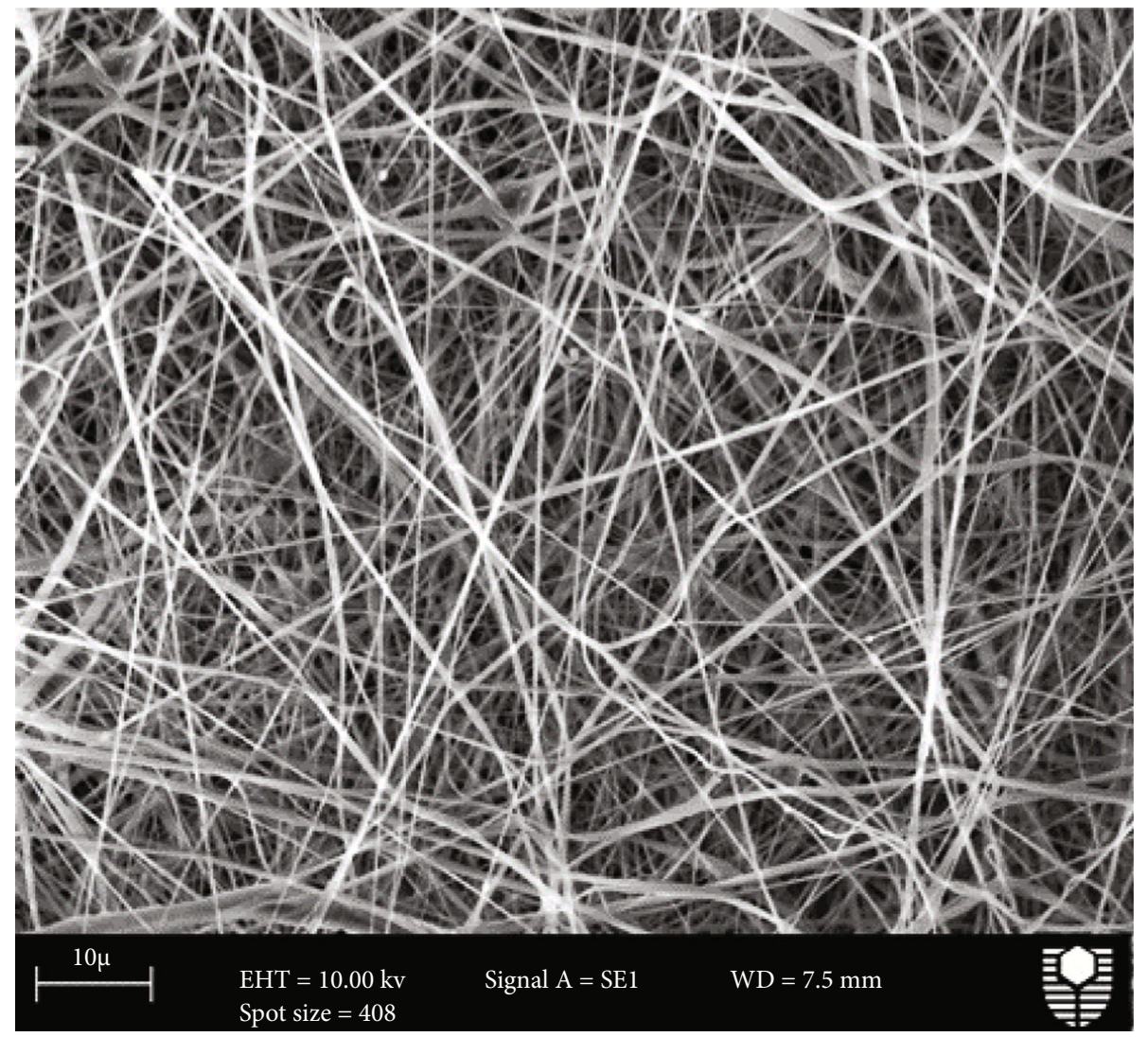

(e)

FIgURe 1: Continued. 


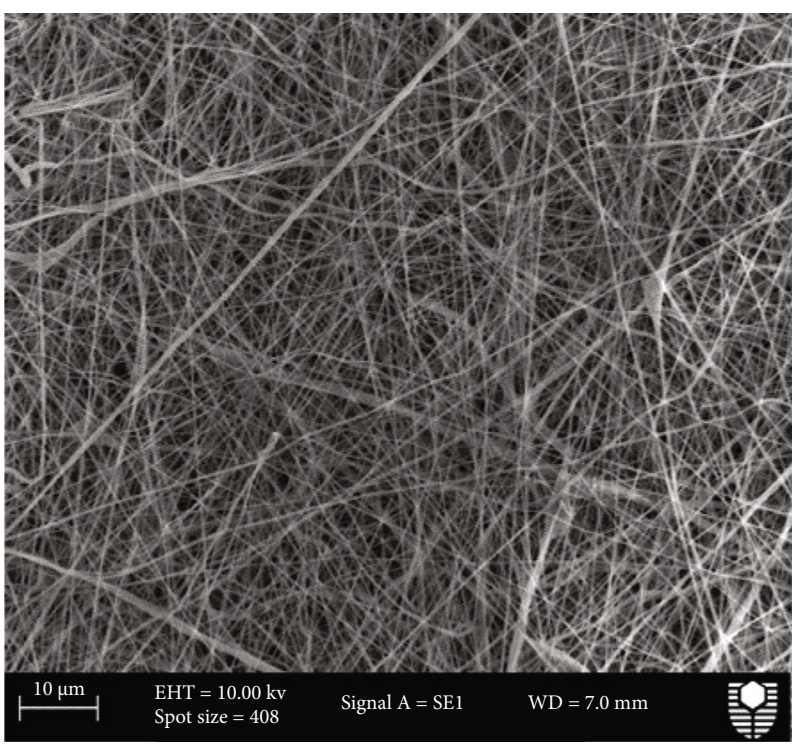

(f)

FIgure 1: SEM micrographs of electrospun nanofibres: (a) PLA, (b) PLA/TCH, (c) PCL, (d) PCL/TCH, (e) PCL/MPs, and (f) PCL/MPs/ TCH. All scale bars represent $10 \mu \mathrm{m}$.

TABLE 1: Summaries of average nanofibre diameters for all electrospun nanofibre membrane structures.

\begin{tabular}{lcccccc}
\hline & PLA & PCL & PCL/MPs & PLA/TCH & PCL/TCH & PCL/MPs/TCH \\
\hline Nanofibre diameter $(\mathrm{nm})$ & $510 \pm 20$ & $368 \pm 15$ & $316 \pm 20$ & $397 \pm 20$ & $279 \pm 15$ & $225 \pm 15$ \\
\hline
\end{tabular}

can be ascribed to amphoteric molecules in TCH drug with several ionisable function groups [31], which in turn leads to increasing the solution electrical conductivity [32].

4.2. FTIR Evaluation. Figure 2 demonstrates the FTIR spectra of PLA, PLA/TCH, PCL, PCL/TCH and PCL/MPs/TCH. The spectra exhibit initially carbonyl stretching $(\mathrm{C}=\mathrm{O})$ band assigned at $1722 \mathrm{~cm}^{-1}$ for PCL nanofibres, as well as at $1750 \mathrm{~cm}^{-1}$ for PLA nanofibres. The associated peaks at 1453 and $1382 \mathrm{~cm}^{-1}$ are indicative of $\mathrm{C}-\mathrm{H}$ deformation in relation to $\mathrm{PLA}$. In addition, many peaks represent $\mathrm{C}-\mathrm{C}$ and $\mathrm{C}-\mathrm{O}$ stretchings in the range from 1240 to $840 \mathrm{~cm}^{-1}$ for PLA and PCL. With respect to PCL/MP composites, it is difficult to indicate the effect of MPs on the bonds, which may be associated with such a small amount of embedded MPs. The spectrum of TCH is also hard to be allocated for the band shift. However, main noticeable change in FTIR spectra lies in the appearance of weak bands of $\mathrm{TCH}$ at 1615 and $1580 \mathrm{~cm}^{-1}$, which are assigned to $\mathrm{C}=\mathrm{O}$ stretching in ring $\mathrm{A}$ and $\mathrm{C}=\mathrm{O}$ stretching in ring $\mathrm{C}$, respectively [32]. This result directly confirms the encapsulation of TCH drug in existence.

4.3. TGA Analysis. TGA curves of PLA, PCL, and PCL/MP electrospun nanocomposite fibre membranes loaded with TCH are presented in Figures 3(a) and (b). The incorporation of TCH drug and MPs do not appear to induce a significant variation in the thermal stability of PCL where the degradation-peak range is between 398 and $401^{\circ} \mathrm{C}$. When $\mathrm{TCH}$ is added to PLA, it has been evidently shown that a peak shift to higher temperatures takes place within fibre membranes in a clear sign of the delay of thermal degradation. The temperature associated with the TGA peak, assigned to PLA fibre membrane at $310^{\circ} \mathrm{C}$, has been found to shift to $348^{\circ} \mathrm{C}$ when loaded with $\mathrm{TCH}$. This phenomenon implies much better thermal stability. Furthermore, the residual masses have been increasingly recorded to be $2.20 \%$ and $4.50 \%$ for PLA and PLA/TCH, respectively, as opposed to $0.01 \%, 3.10 \%, 3.20 \%$ and $3.50 \%$ for PCL, PCL/ TCH, PCL/MPs and PCL/MPs/TCH. The high residual mass of PCL/MPs, when compared with PCL, is associated with the appearance of remaining MP particles. In addition, $\mathrm{PLA} / \mathrm{TCH}$ and $\mathrm{PCL} / \mathrm{TCH}$ composites give rise to higher char residues as opposed to PLA and PCL. Such a phenomenon could mean that there are complicated interactions and synergistic effects in the material system with respect to char residue formation [33] among PLA/TCH and PCL/TCH composites.

4.4. Thermal Properties. Thermal properties of electrospun fibres with MPs and TCH drugs are shown in Figure 4 and Table 2. The glass transition temperature $\left(T_{g}\right)$ of PCL within fibre composites decreases remarkably while there was a slight decline in melting temperature $\left(T_{m}\right)$ with the inclusion of MPs. The decrease in $T_{g}$ can be attributed to the effect of embedded MPs with a typical anisotropic characteristic, which is in good accordance with previous work [34]. The addition of TCH in possession of a low molecular weight inevitably yields a decrease in $T_{g}$ and $T_{m}$ values for 


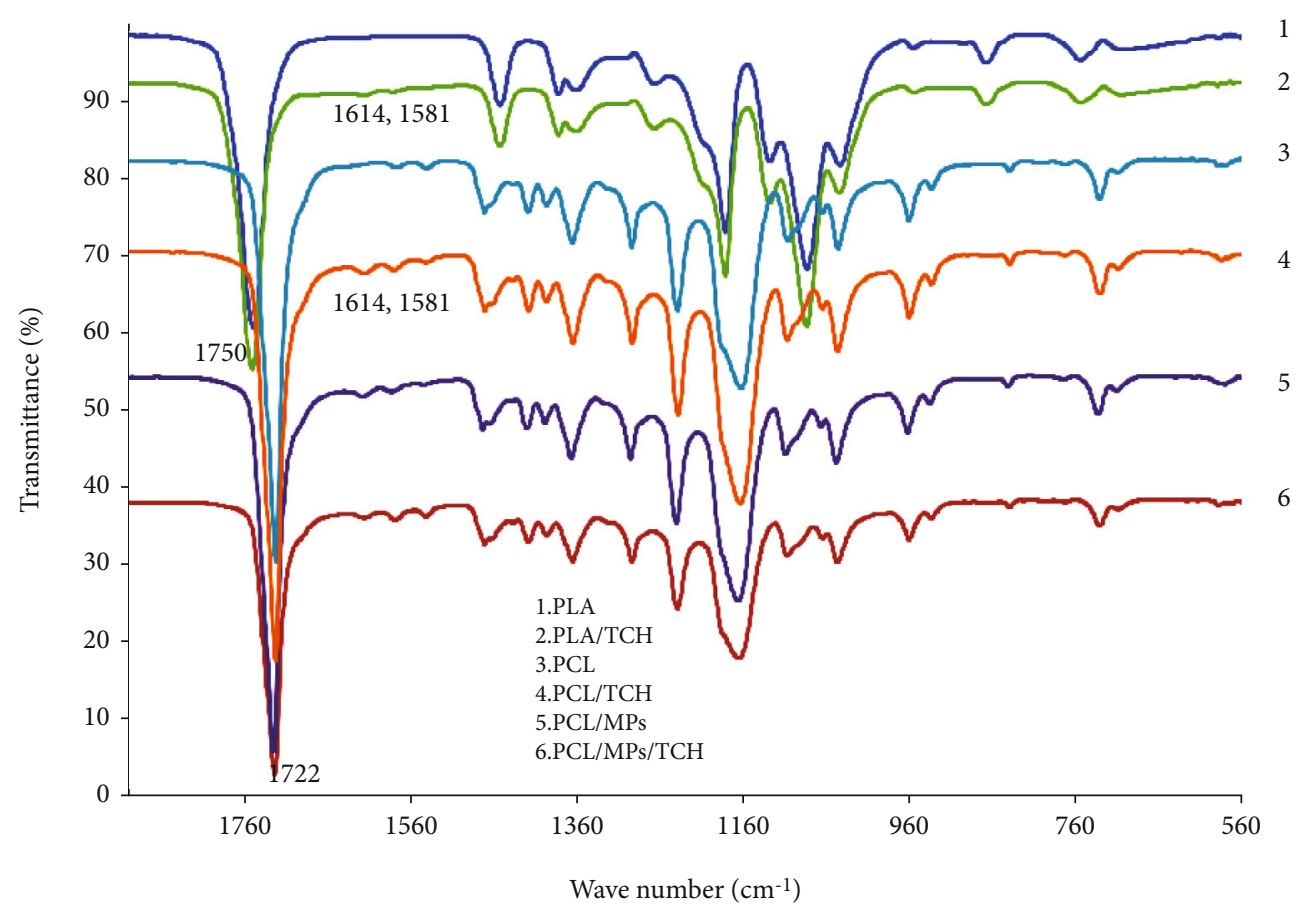

FIGURE 2: FTIR spectra of selected electrospun nanofibre membrane samples demonstrating relative FTIR peaks.

both PCL and PLA. TCH molecules have short chains, which results in reducing the packing density of polymeric chains, and further facilitates the chain mobility with a clear sign of lower $T_{g}$.

4.5. Crystallinity Level. The crystalline structures of composites are presented in Figure 5 with respect to XRD patterns. The peaks were labelled for the XRD reflection, and the crystal planes $(h k l)$, corresponding to main crystalline peaks, were detected in the DICVOL program of the FullProf software. The XRD patterns of PCL possess two diffraction peaks at corresponding angles of $2 \theta=$ $21.4^{\circ}$ and $23.8^{\circ}$ without any considerable difference in the peak position. This suggests that the addition of MPs and $\mathrm{TCH}$ has very minor impact on the alteration of crystalline structures in electrospun composite nanofibres. As such, TCH may be dispersed in an amorphous state within nanofibre membranes. In addition, the large surface area combined with small nanofibre diameters arising from the electrospinning process can promote quick solvent evaporation, thus resulting in a short period for drug recrystallisation along with the creation of the preferred configuration in an amorphous state [35].

The XRD results were useful to identify the degree of crystallinity $X_{c}$ in Table 2 . It is revealed that the $X_{c}$ of PCL decreases considerably when incorporated with MPs. This decreasing trend might be explained by abundant nucleation cores to generate the proliferation of tiny crystallites leading to an overall low degree of crystallinity [36]. MPs seem to greatly accelerate the nucleation process. Consequently, this phenomenon results in a shorter period for the disentanglement of molecular chains. The associated degree of crystallinity is usually induced by the restricted mobility of polymeric chains, which further hinders good crystal growth. The inclusion of TCH within PLA, PCL, and PCL/ MP nanocomposites could lead to a moderate reduction in the degree of crystallinity. The presence of $\mathrm{TCH}$ appears to significantly accelerate the nucleation process. As a result, the disentanglement of molecular chains takes less time due to this occurrence.

4.6. In Vitro Drug Release. TCH release profiles from electrospun PLA, PCL and PCL/MPs composite nanofibre membranes are illustrated in Figure 6. Similarly, both PCL and PCL/MPs material samples show a fast drug release behaviour. In the initial $25 \mathrm{~min}, 66 \%$ and 58\% drugs were released from PCL samples and PCL/MPs accordingly relative to $34 \%$ from PLA. The initial higher TCH release from PCL nanofibres accounts for a relatively high $X_{c}$ of PCL/TCH at $59.1 \%$, as opposed to $53.7 \%$ and $11.3 \%$ for PCL/MPs/TCH and PLA/TCH, respectively, according to Table 2 . The high crystallinity of nanofibres easily restricts the mobility of drug molecules to remain on the fibre surface areas. In addition, rapid drug release is associated with the electrospinning process, which includes quick solvent evaporation and highly ionic interactions leading to the mobility of drug molecules on the surfaces of electrospun fibres. Nevertheless, the $\mathrm{TCH}$ release from PCL starts to become slower than that from PCL/MPs nanofibres after $25 \mathrm{~h}$. The incorporation of MPs leads to an increase in fibre porosity [30], thus resulting in the release acceleration of TCH. In addition, the small diameters of fibrous structures obtained from PCL/MPs/ $\mathrm{TCH}$, when compared with PCL/TCH, tend to cause the increase in surface area, thus leading to short diffusion distance with fast $\mathrm{TCH}$ release. On the other hand, $\mathrm{TCH}$ release from PLA intends to become faster than those obtained 


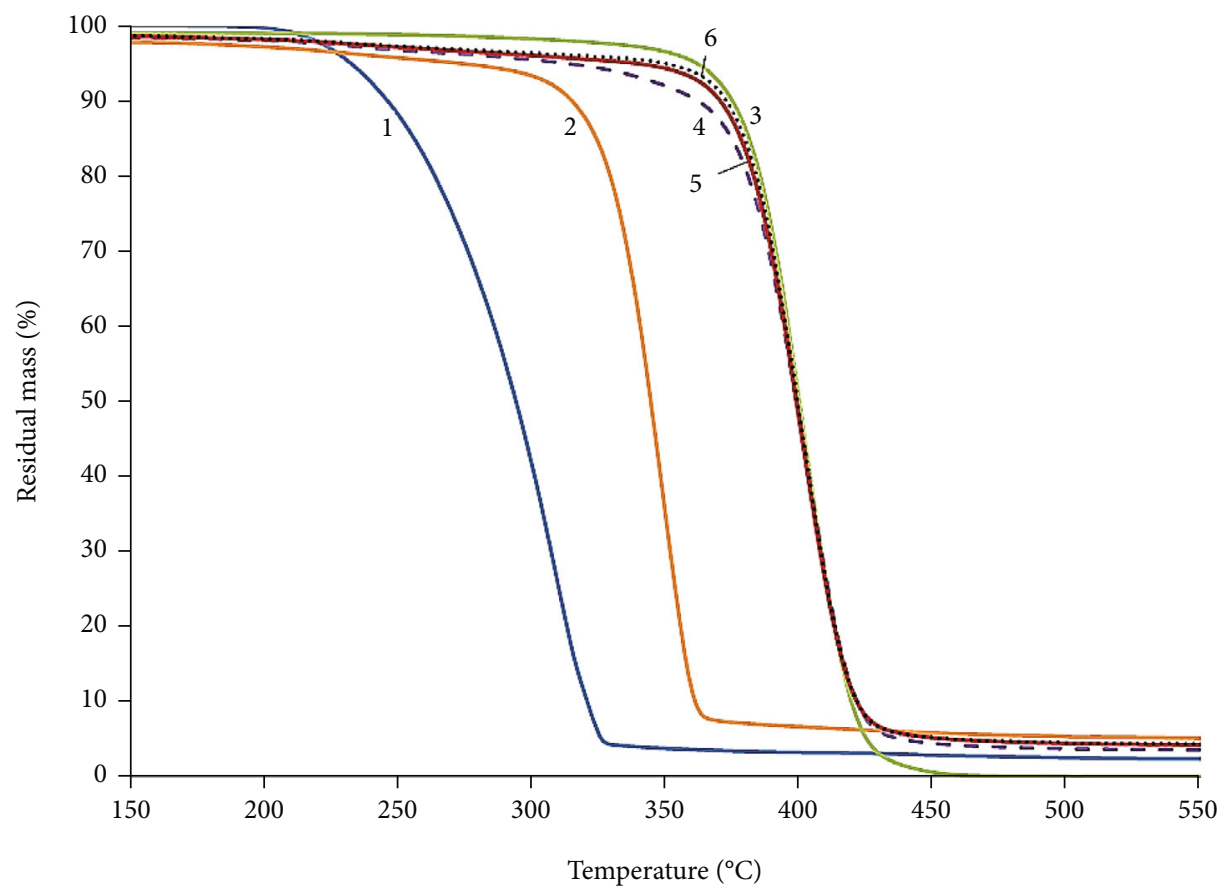

(a)

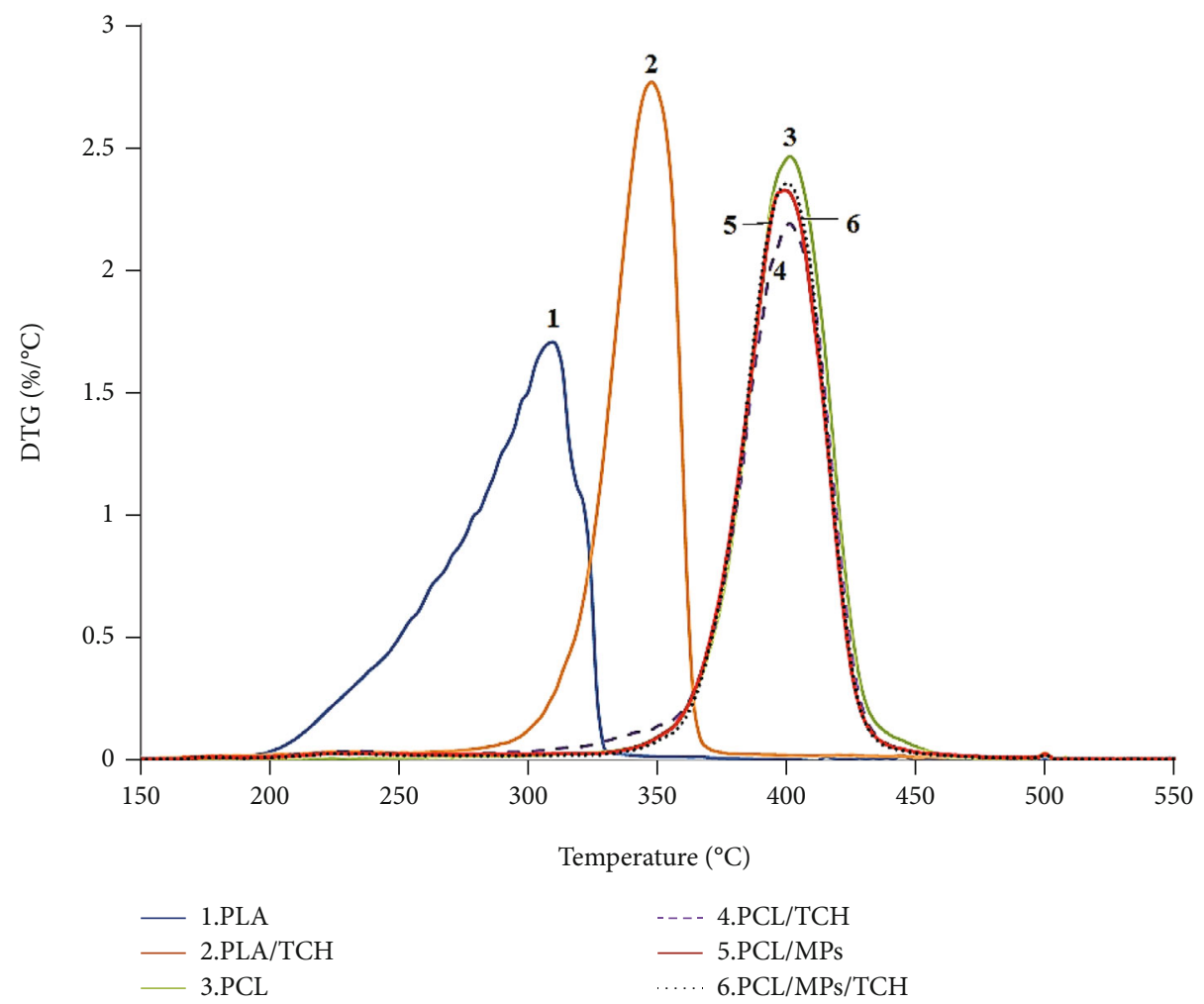

(b)

Figure 3: (a) TGA curves and (b) DTGA curves for typical PLA, PCL, and PCL/MPs fibre membranes.

from PCL and PCL/MPs nanofibres after 50 and $110 \mathrm{~h}$, respectively. Over the long term of drug release, semicrystalline PCL can delay the water diffusion inside nanofibres, and accordingly the absorption of water happens slowly when compared with PLA.
4.7. Release Kinetics. The release kinetics of $\mathrm{TCH}$ drug was investigated by fitting Zeng model [29] to experimental release data, as demonstrated in Figure 7. The addition of MPs to PCL gives rise to a minor decrease in $\Delta G$ values from $4.94 \times 10^{-21}$ to $4.19 \times 10^{-21} \mathrm{~J}$, indicating that there is a slight 


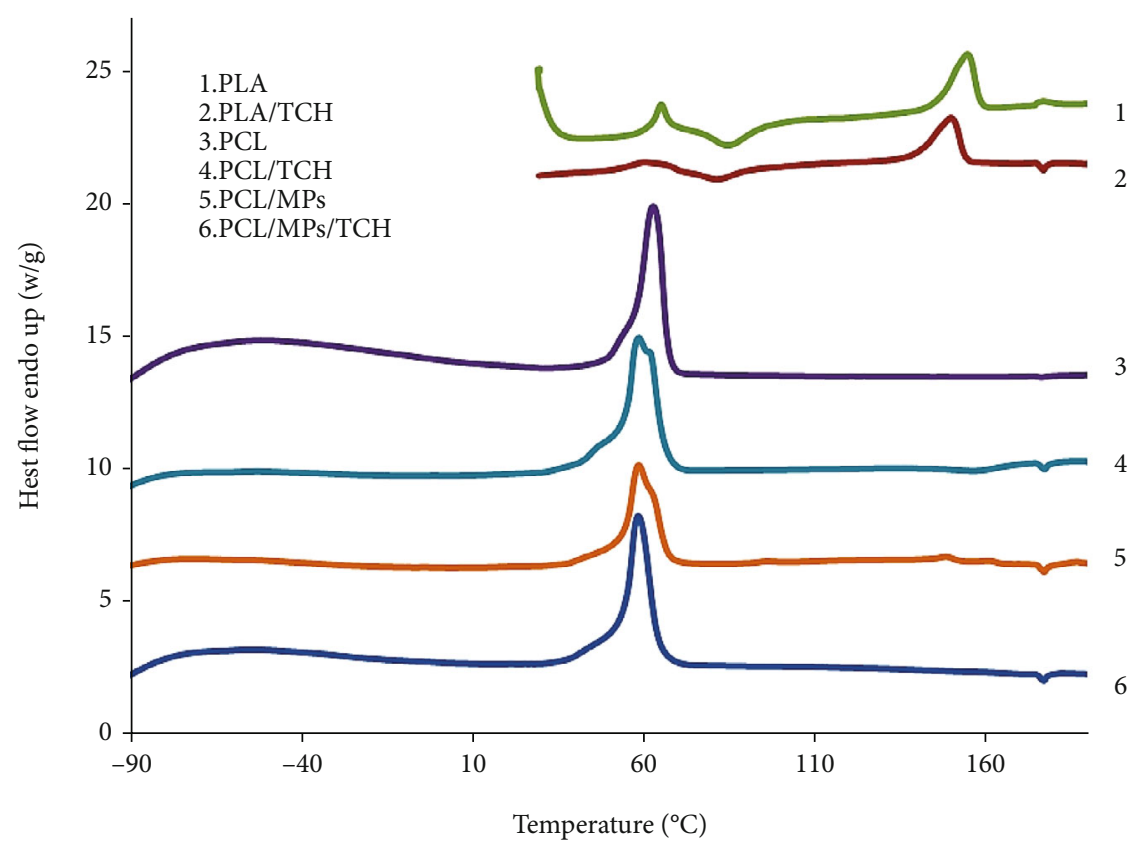

Figure 4: DSC thermograms for selected PLA and PCL-based material samples.

TABLE 2: Thermal properties of PLA, PCL, their blends and composites.

\begin{tabular}{|c|c|c|c|c|c|c|}
\hline Material sample & $T_{g}\left({ }^{\circ} \mathrm{C}\right) \mathrm{PCL}$ & $T_{m}\left({ }^{\circ} \mathrm{C}\right) \mathrm{PCL}$ & $T_{g}\left({ }^{\circ} \mathrm{C}\right)$ PLA & $T_{c}\left({ }^{\circ} \mathrm{C}\right)$ PLA & $T_{m}\left({ }^{\circ} \mathrm{C}\right)$ PLA & $X_{c}$ \\
\hline PLA & & & 62.1 & 87.2 & 155.6 & 12.6 \\
\hline $\mathrm{PLA} / \mathrm{TCH}$ & & & 54.8 & 82.4 & 150.2 & 11.3 \\
\hline PCL & -56.2 & 63.4 & & & & 64.6 \\
\hline $\mathrm{PCL} / \mathrm{TCH}$ & -58.9 & 58.9 & & & & 59.1 \\
\hline PCL/MPs & -57.2 & 59.0 & & & & 54.2 \\
\hline PCL/MPs/TCH & -60.0 & 58.7 & & & & 53.7 \\
\hline
\end{tabular}

Note: Calculations were based on the average data obtained from the three sets of samples. The standard deviations for $T_{c}, T_{g}$, and $T_{m}$ values were less than $0.5 \% . X_{c}$ : degree of crystallinity.

enhancement in the interaction between PCL nanofibres and $\mathrm{TCH}$. However, such an increase adjusts the $\mathrm{TCH}$ release at the burst stage, and the drug release from PCL/MPs/TCH was faster than that from PCL/TCH after $25 \mathrm{~h}$. This perception may be ascribed to the formation of small fibre diameters with the addition of MPs to accelerate the TCH release. Moreover, increasing $K_{s}$ values reflects the enlargement in fibre pore size, which in turn leads to fast $\mathrm{TCH}$ release. On the other hand, PLA/TCH yields a lower $\Delta G$ than PCL/ TCH $\left(4.94 \times 10^{-21} \mathrm{~J}\right.$ vs. $\left.2.0 \times 10^{-21} \mathrm{~J}\right)$, which suggests that PLA partially decreases the release rate of TCH due to their improved interaction. The $\mathrm{TCH}$ release kinetic is not completely interpreted by Zeng model because it does not take into account the erosion/biodegradation and dimensional alteration of nanofibres. This is evidenced by the model compatibility with $\mathrm{PCL} / \mathrm{TCH}$, which has less mass loss as opposed to PLA/TCH and PCL/MPs/TCH.

4.8. Mass Loss of Fibre Membranes. The biodegradation behaviour of nanofibre membranes is shown in Figure 8. After the degradation in PBS for the first 3 to $24 \mathrm{~h}$, the weights of PCL/TCH and PCL/MPs/TCH nanofibre membranes remain nearly unchanged within standard errors. Nonetheless, there was a high initial mass loss for PLA/ TCH nanofibre membranes (i.e., $3 \%$ for PLA, $0.2 \%$ for PCL and $0.3 \%$ for PCL/MPs). Mass loss for PCL and PCL/ MPs becomes more pronounced after $72 \mathrm{~h}$, and it increases over the degradation time of $336 \mathrm{~h}$ to reach $12.5 \%$ for PLA, $1.8 \%$ for PCL and $2.9 \%$ for PCL/MPs accordingly. Since semicrystalline PCL may hinder the water penetration inside the nanofibres, water absorption occurs more slowly when compared with amorphous PLA. Moreover, the low crystallinity of PLA can accelerate the fibre degradation. The amorphous regions can easily degrade as opposed to the crystalline regions due to random and less tightly packed arrangement of molecular chains. On the other hand, the surface areas exposed to the PBS are larger in the case of embedding MPs due to resulting small nanofibre diameters and also the increase in fibre porosity [30] at the nanofibre surfaces with the addition of MPs.

Figure 9 shows the morphological variations in electrospun nanofibres after the immersion in PBS over a two- 


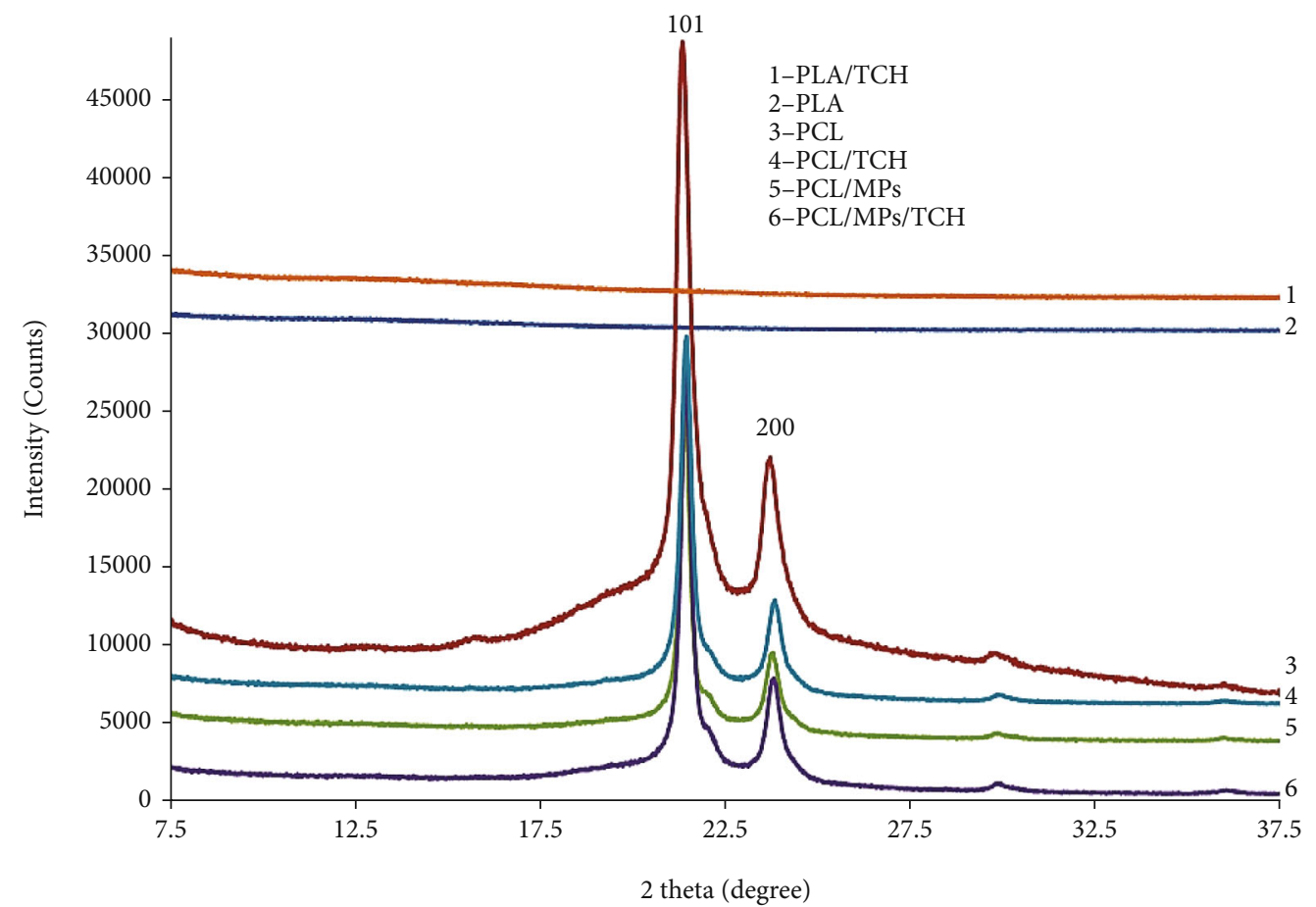

FIGURE 5: XRD patterns for selected material samples showing the relative positions of reflection peaks when incorporated with MPs and TCH.

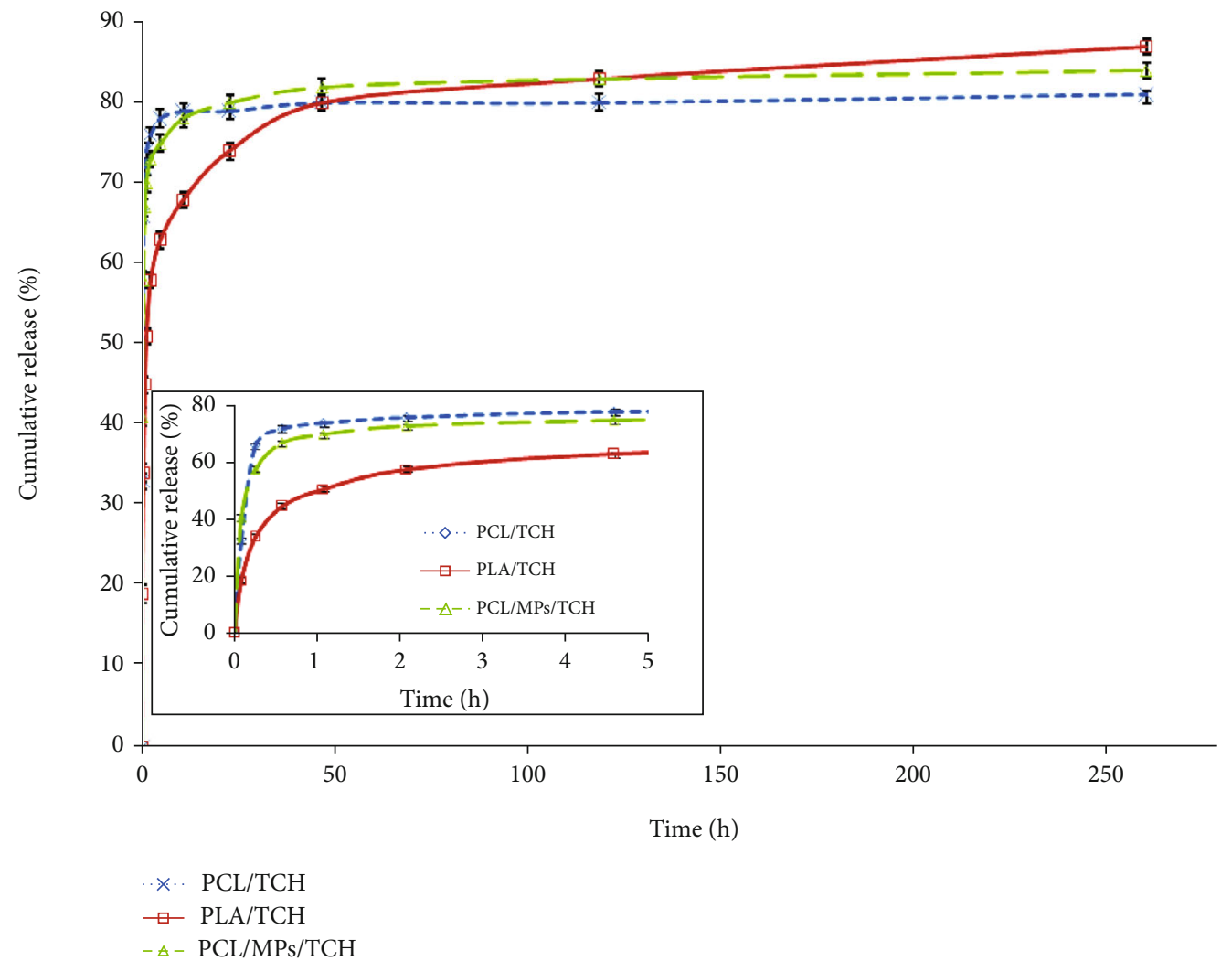

Figure 6: TCH release profiles from PLA, PCL and PCL/MPs.

week period. It has been found that different morphological structures take place for electrospun nanofibre membranes with various nanocomposite formulations. Nanofibre diam- eters increase and the interspaces between electrospun nanofibres drop after $24 \mathrm{~h}$ for PLA/TCH and 7 days for PCL/TCH and PCL/MPs/TCH. The electrospun nanofibres possess 

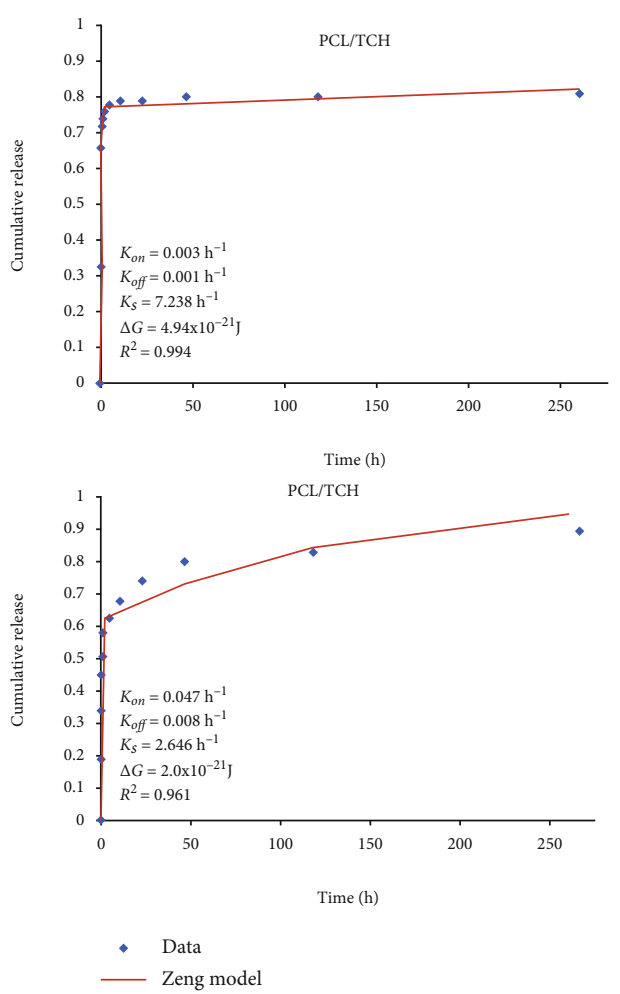

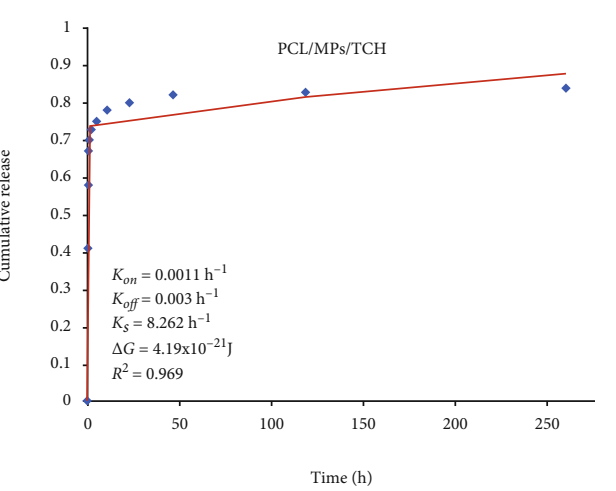

Time (h)

FIGURE 7: Model fitting with TCH release experimental data based on electrospun PCL/TCH, PCL/MPs/TCH, and PLA/TCH nanofibres.

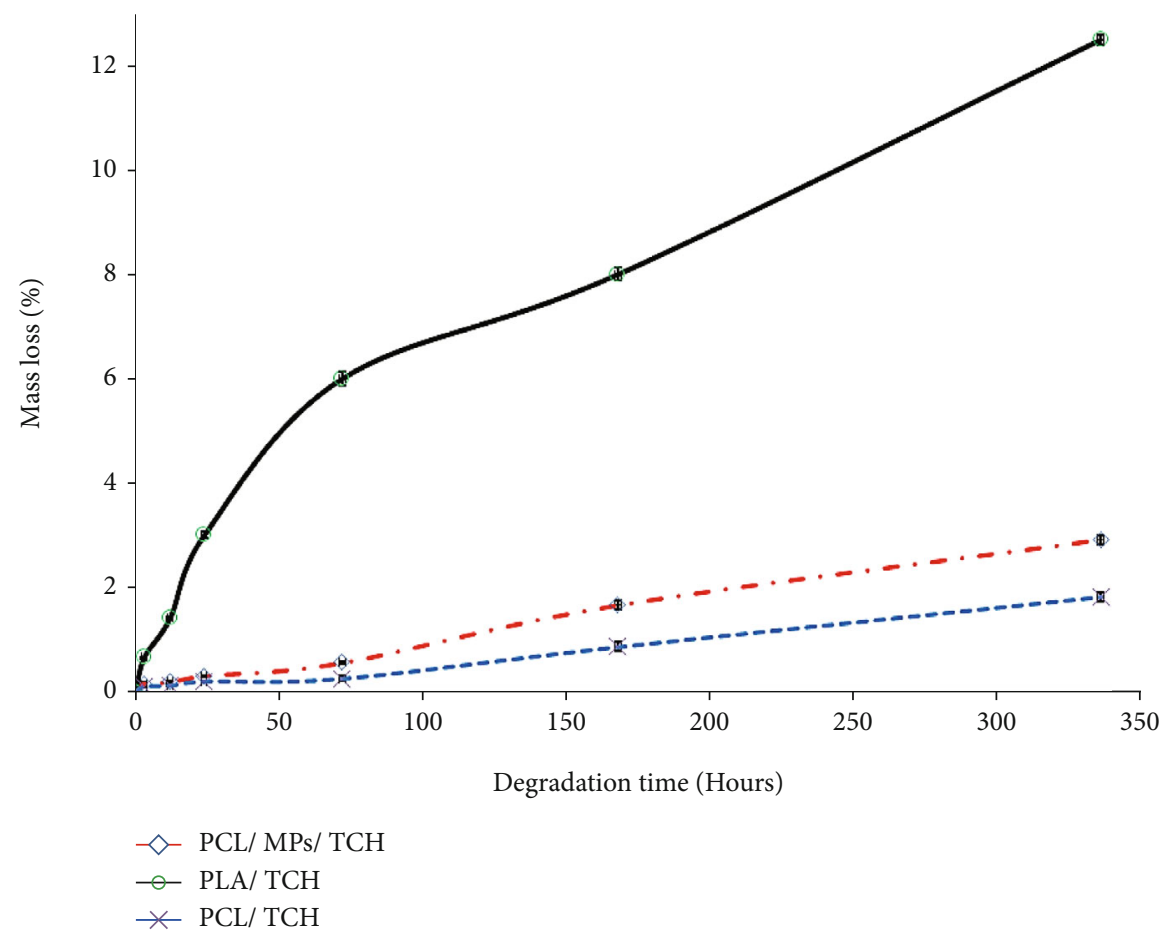

FIgURE 8: Mass loss of electrospun nanofibre membranes during the degradation time.

more wrinkled structures after 7 days when compared with those for initial degradation over $3 \mathrm{~h}$. The degradation firstly takes place in the amorphous area of blend nanofibres and then occurs in the crystalline area. Moreover, the degrada- tion at the nanofibre surfaces could be slower than in nanofibre cores due to carboxylic acid groups existing in the fibre core areas by an ester hydrolysis. These results coincide with those obtained from in vitro drug release. The rapid release 


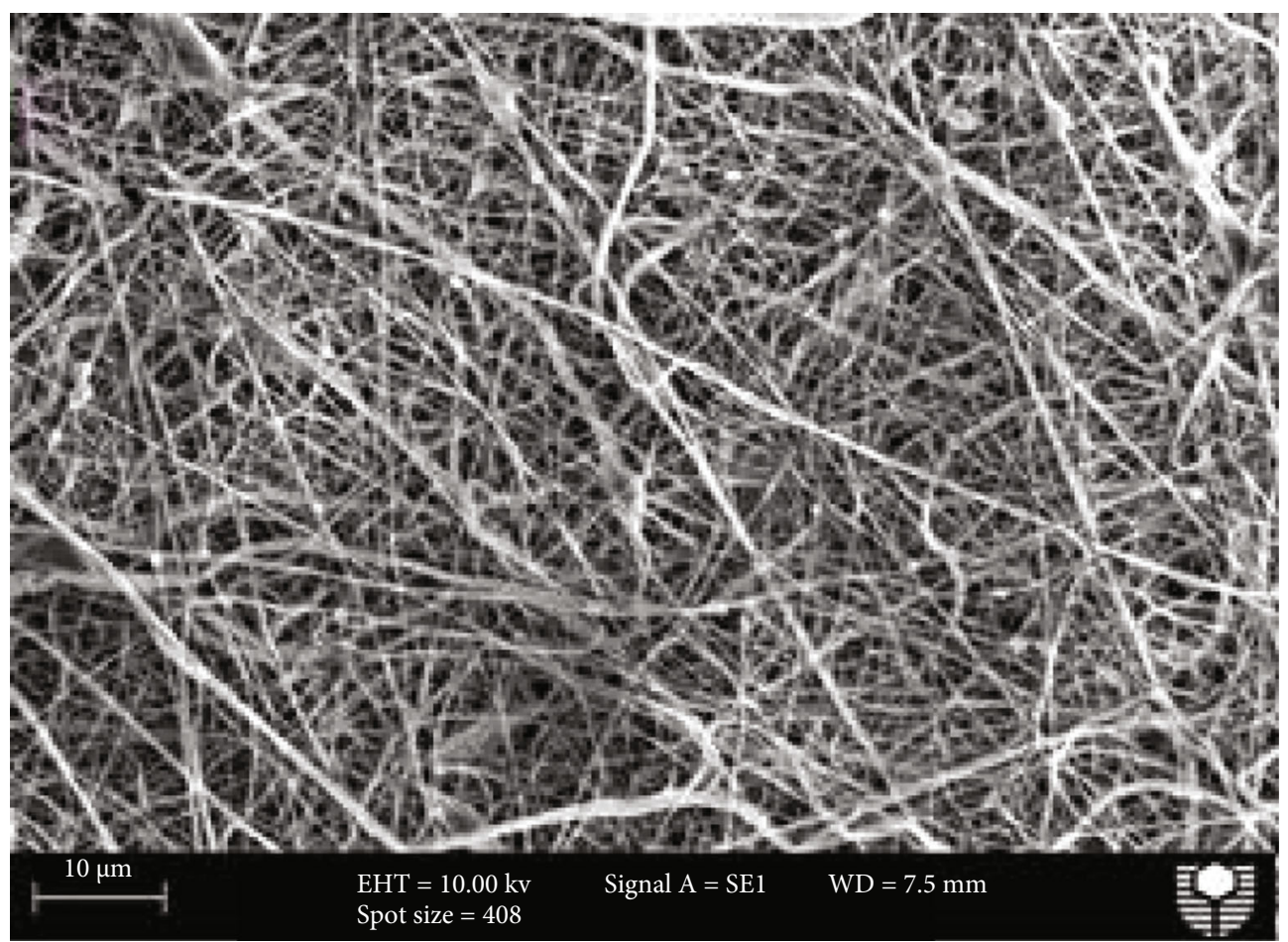

(a)

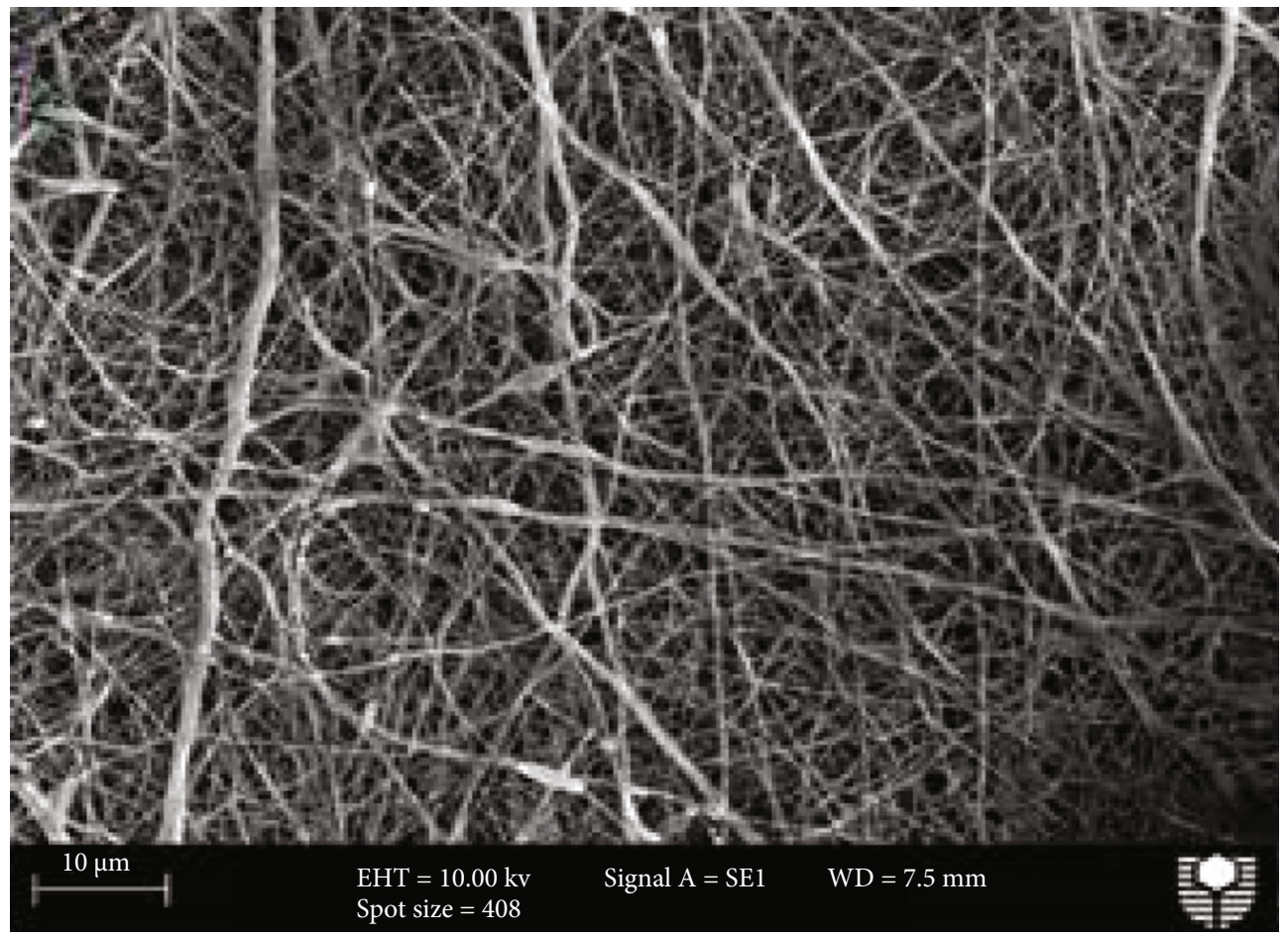

(b)

Figure 9: Continued. 


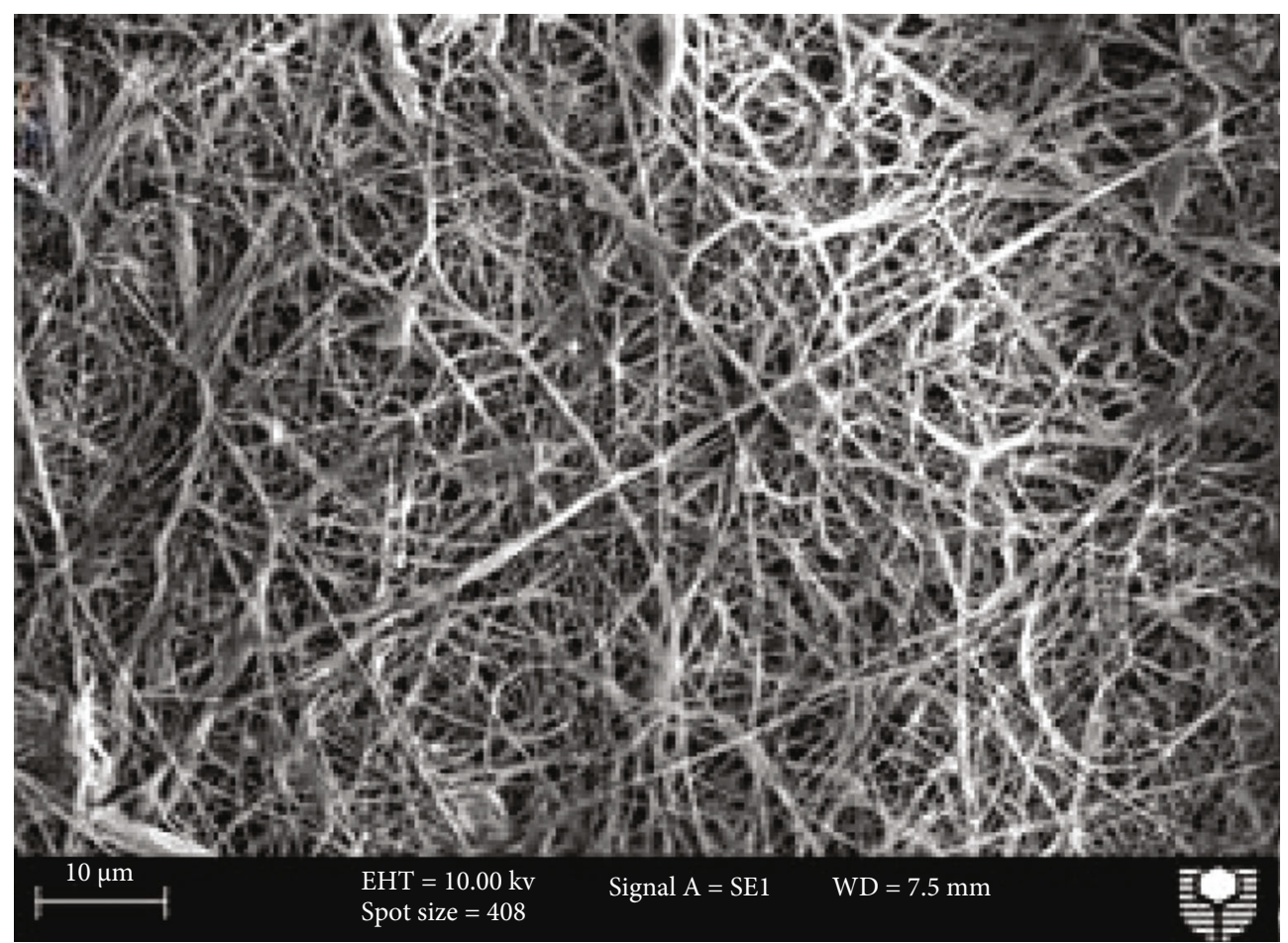

(c)

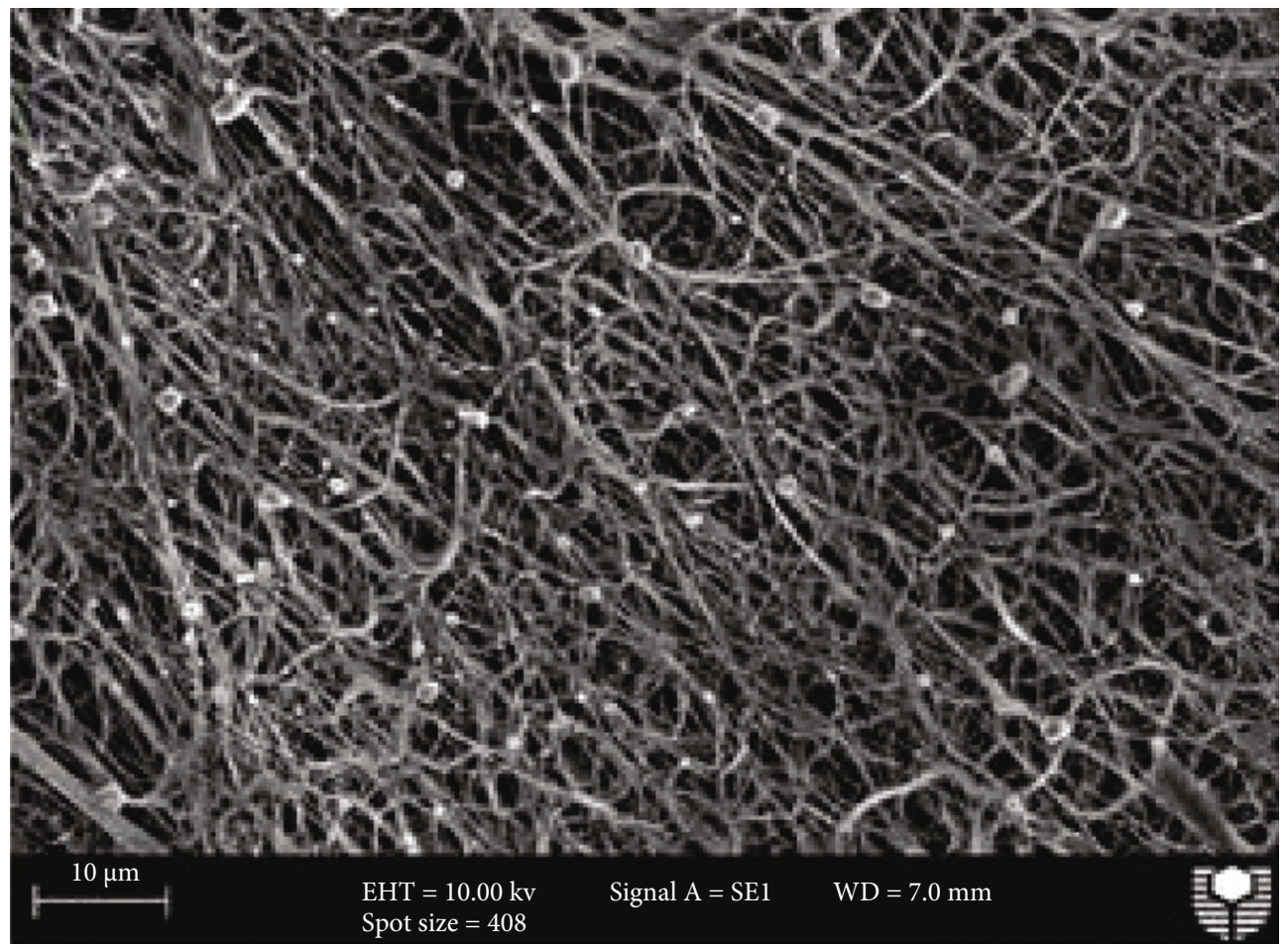

(d)

Figure 9: Continued. 


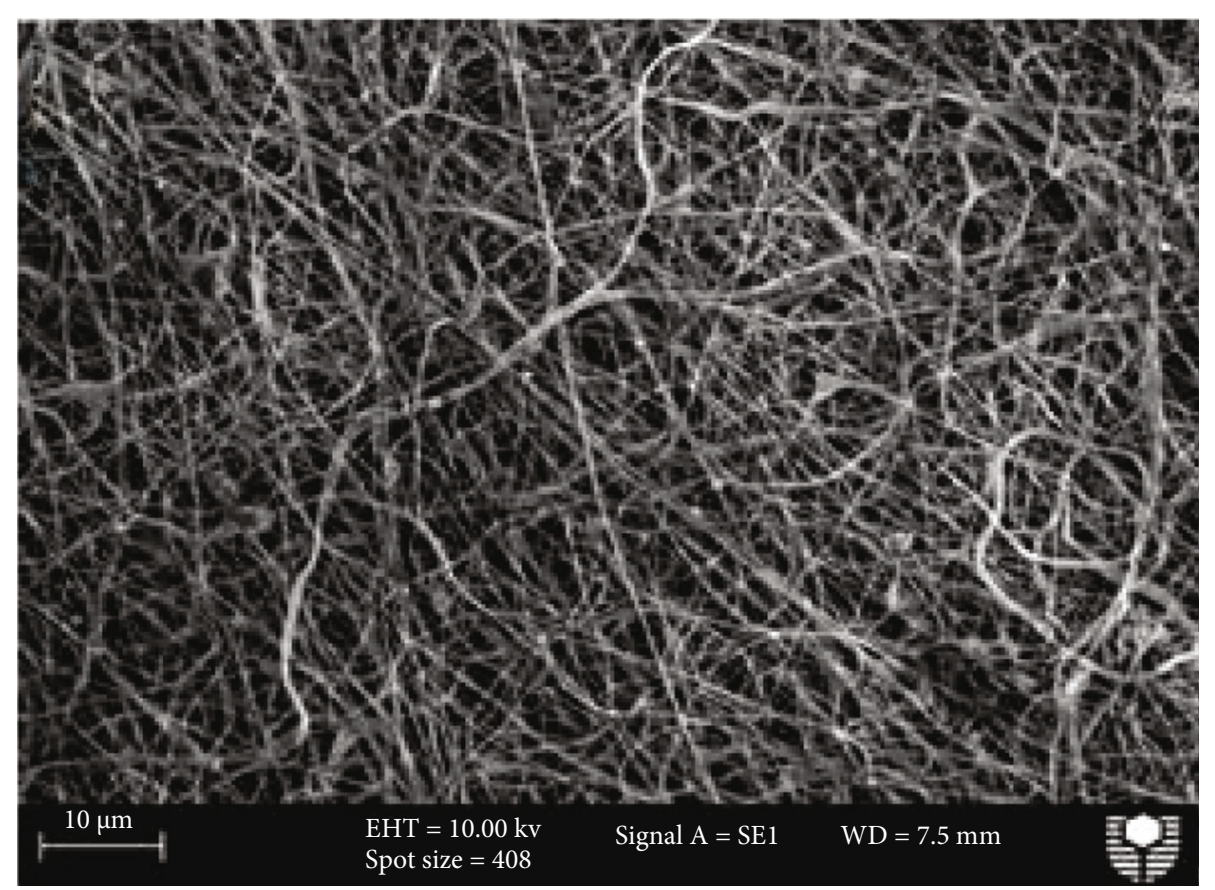

(e)

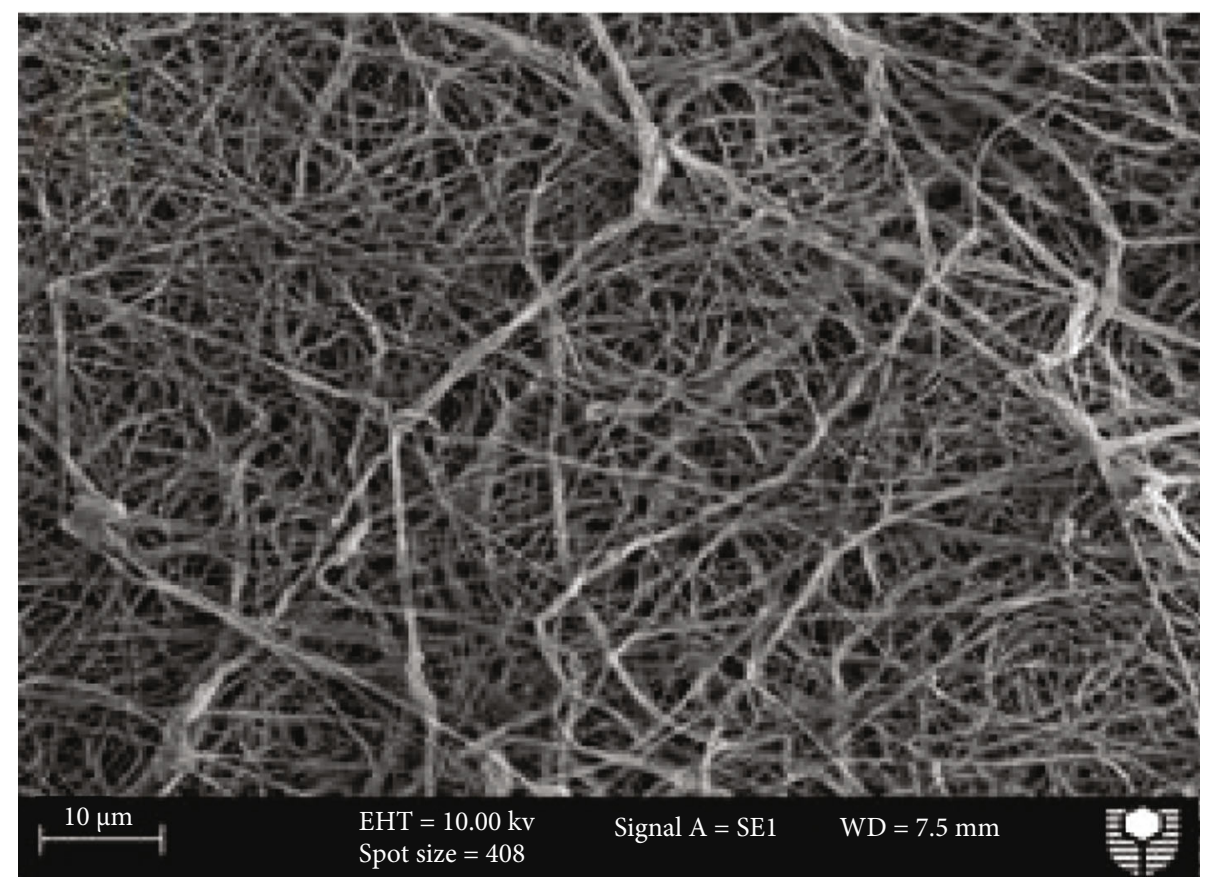

(f)

Figure 9: Continued. 


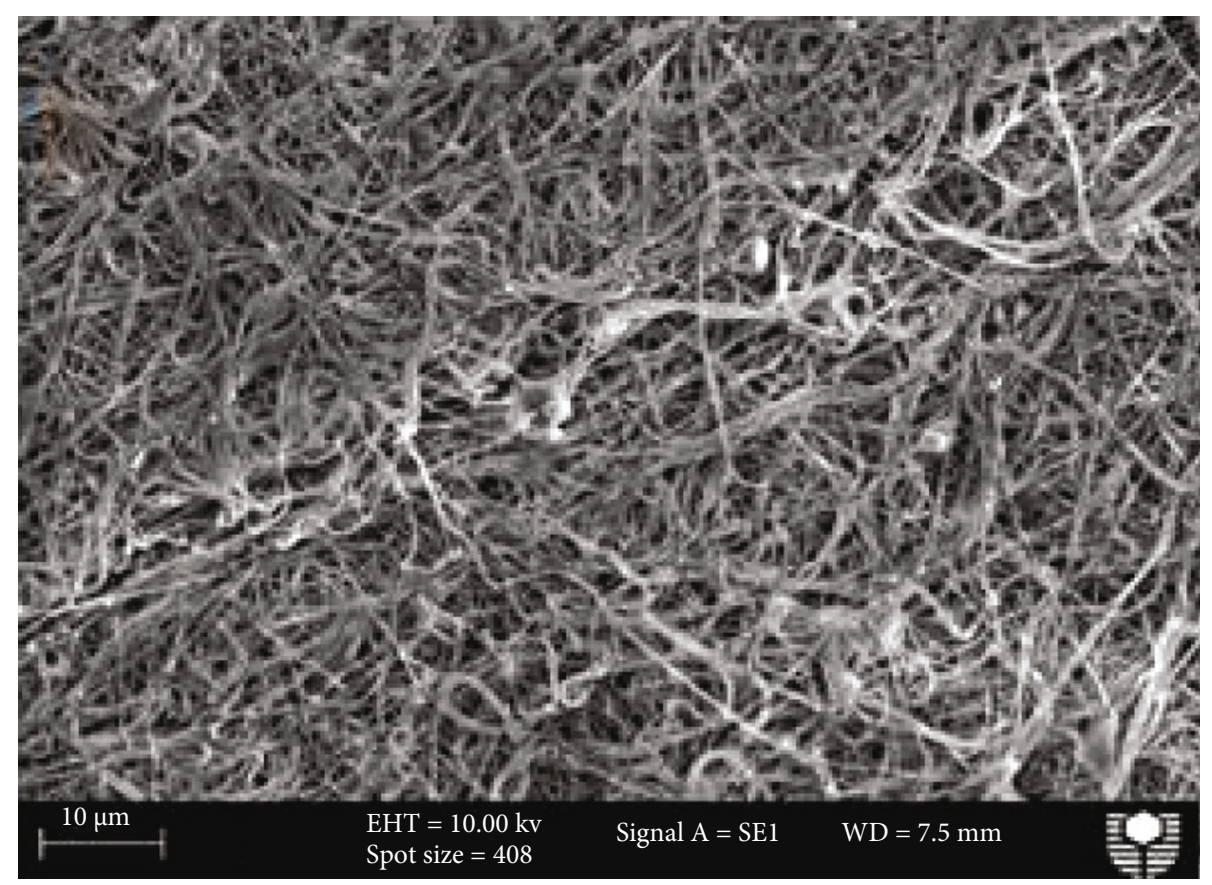

(g)

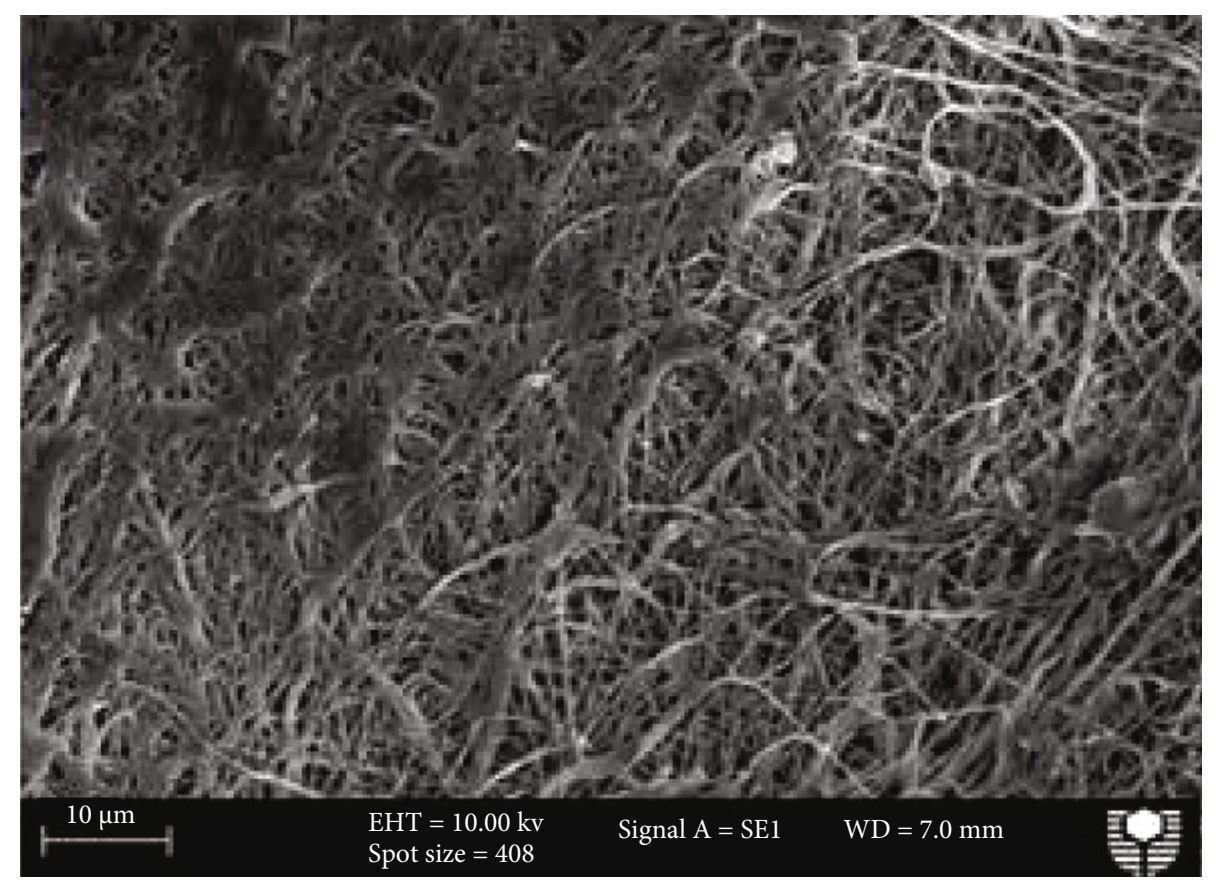

(h)

Figure 9: Continued. 


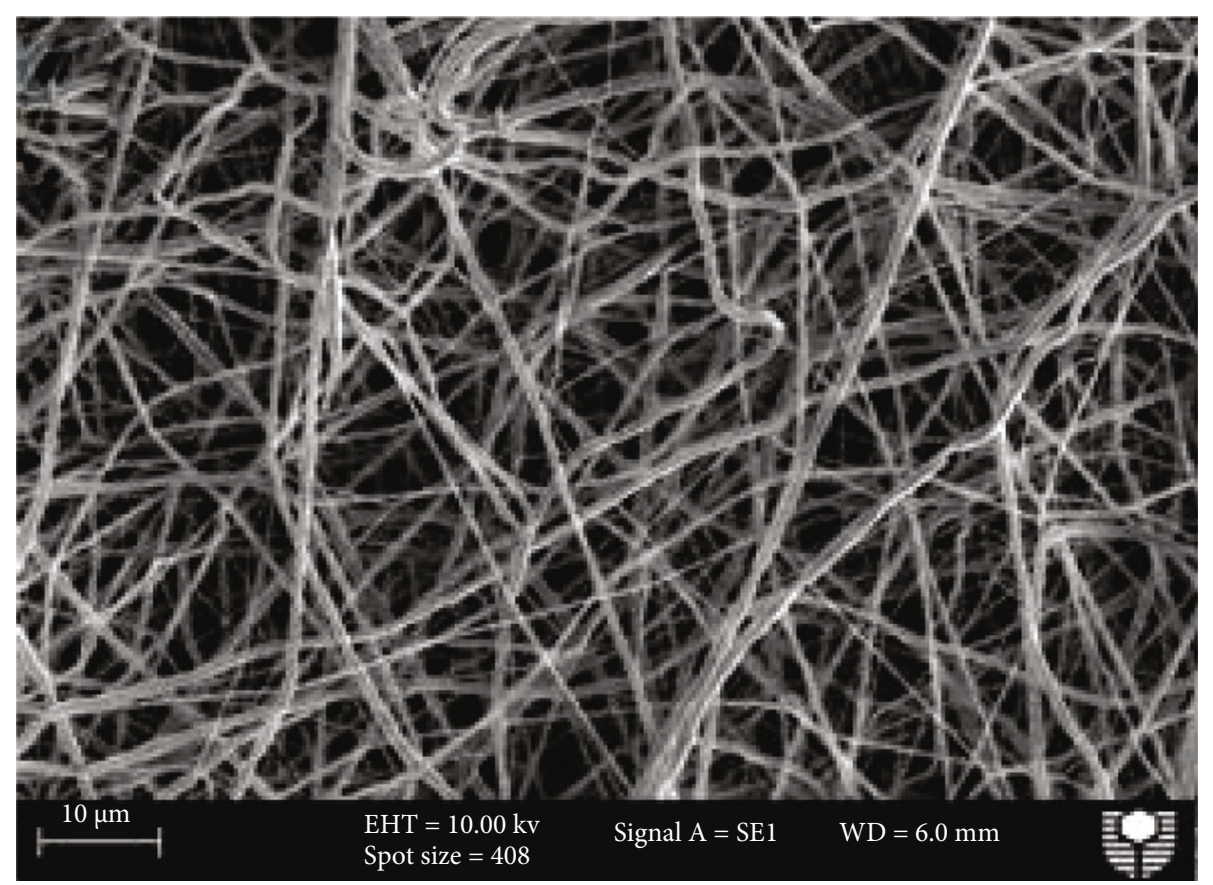

(i)

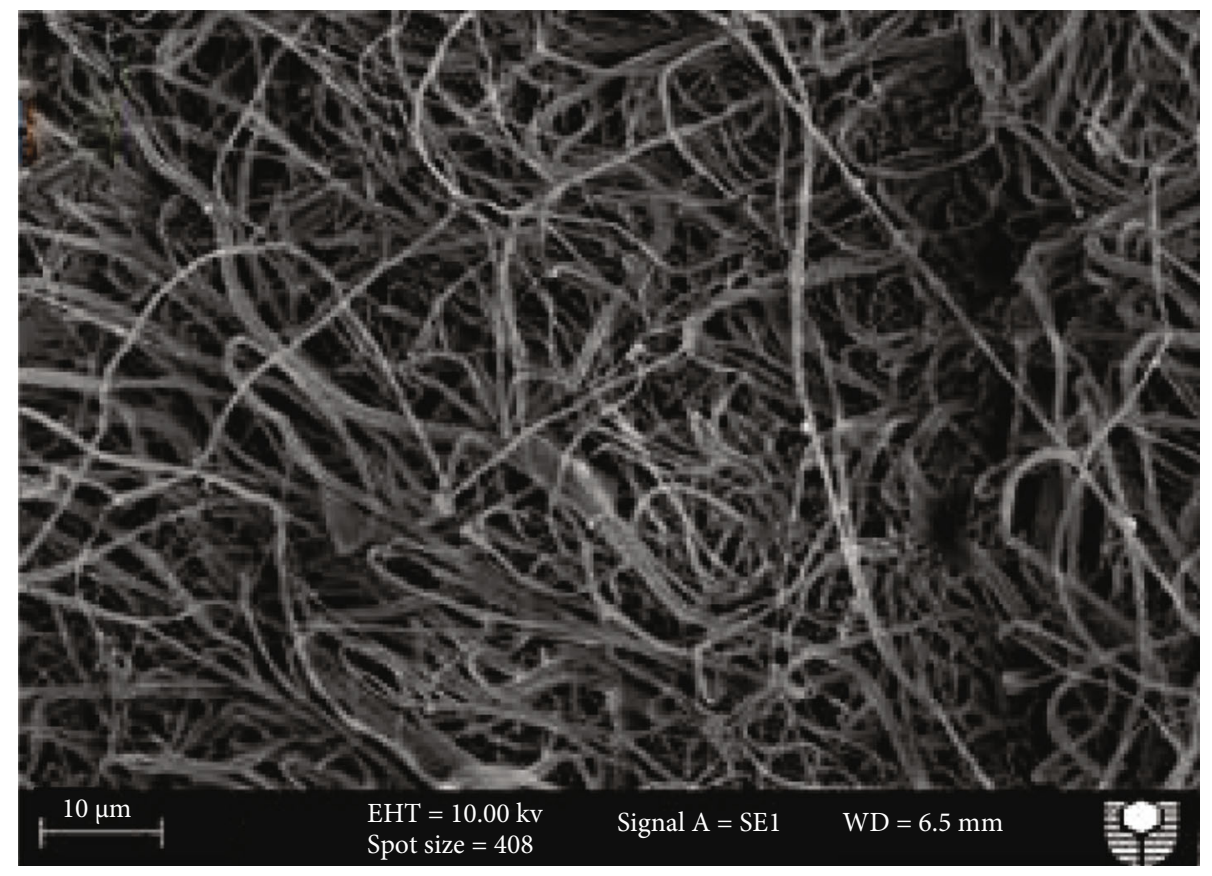

(j)

Figure 9: Continued. 


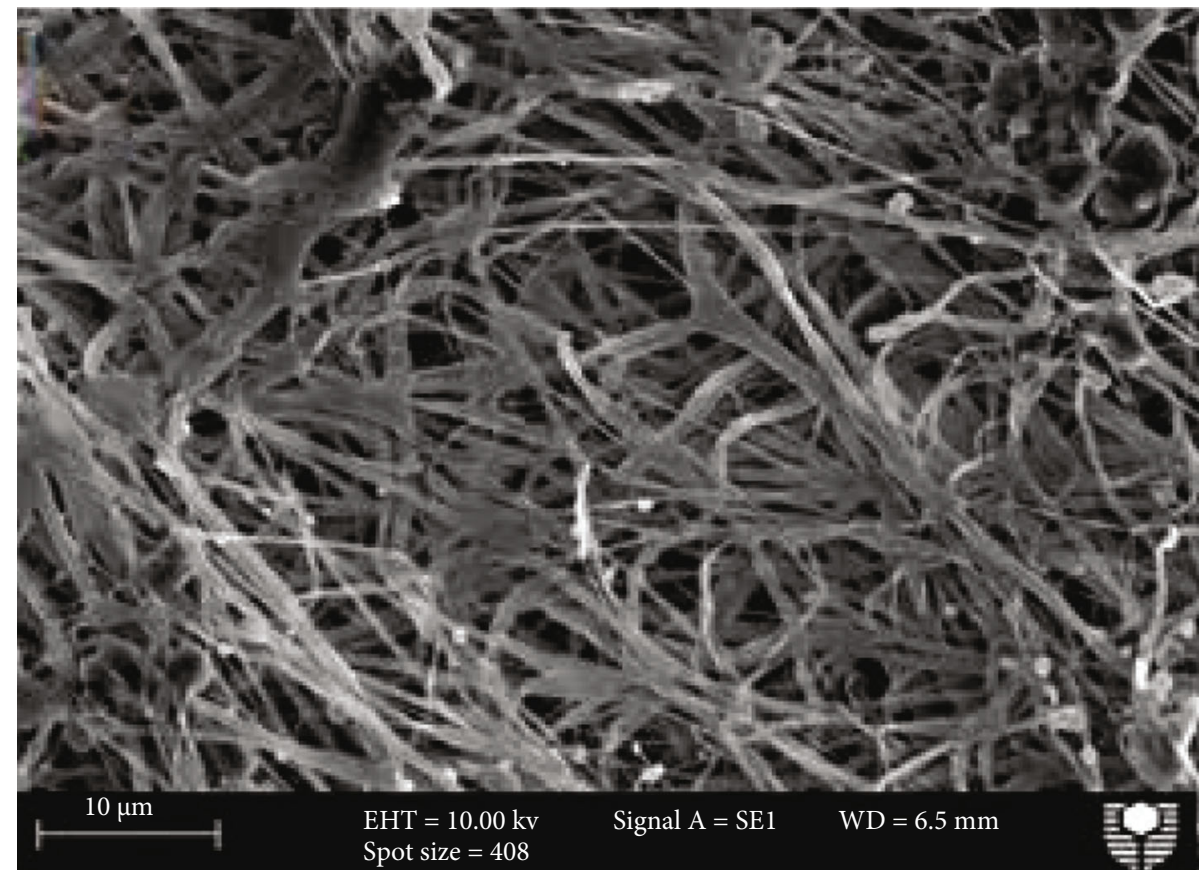

$(\mathrm{k})$

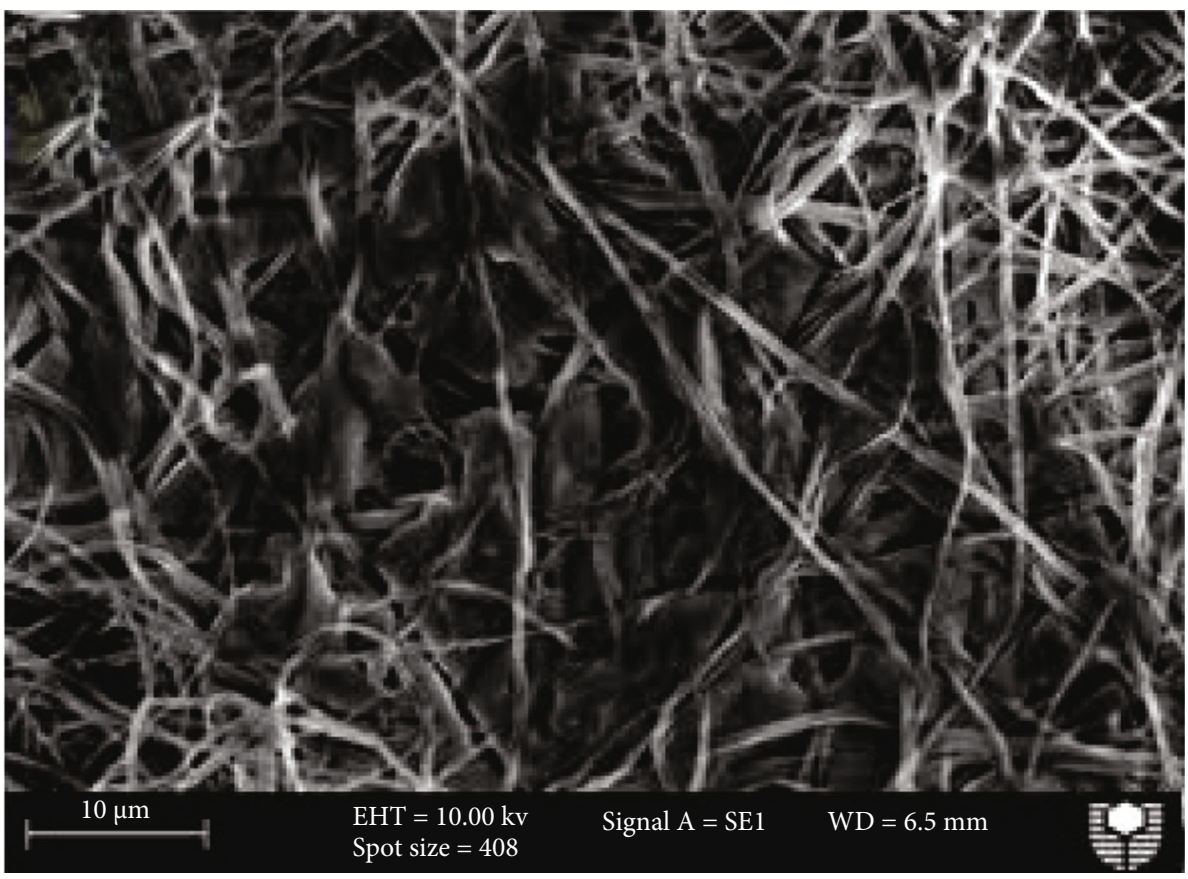

(1)

FIgURE 9: SEM micrographs of in vitro degradation of electrospun nanofibre membranes: (a) to (d) PCL/TCH, (e) to (h) PCL/MPs/TCH, (i) to (l) PLA/TCH for periods of $3 \mathrm{~h}, 24 \mathrm{~h}, 7$ days and 14 days, respectively.

of TCH from PCL can be well compared with PLA over the short-term release before $50 \mathrm{~h}$, after which reversal release would further happen.

\section{Conclusions}

The addition of MPs to PCL and TCH drug enhances the electrical conductivity of a solution, thus leading to the reduction of fibre diameters. FTIR spectra demonstrate a successful encapsulation of TCH within the fibre membranes. When TCH and MPs are utilised to PCL, TGA curves indicate little effect on thermal stability. The addition of TCH to PLA slowed down the rate of thermal degradation. As the MP and TCH were added to PCL, both $T_{g}$ and $T_{m}$ of PCL decreased accordingly. After the MPs were embedded to PCL, $X_{c}$ was reduced as well. In particular, with the incorporation of TCH into PLA, PCL and PCL/MP nanofibre membranes, $X_{c}$ appeared to 
decrease moderately. It may be the case that $\mathrm{TCH}$ becomes scattered in nanofibre membranes in an amorphous state. Electrospun PCL and PCL/MPs nanofibres show a fast drug release behaviour when compared with PLA nanofibres after $25 \mathrm{~min}$. TCH release from PCL nanofibres intended to be slower than that from PCL/ MPs nanofibres after $25 \mathrm{~h}$. In comparison, the TCH release from PLA appeared to be faster than those obtained from PCL and PCL/MPs nanofibres after 50 and $110 \mathrm{~h}$, respectively. Zeng model indicates the minor enhancement in the interaction between PCL nanofibres and TCH with the inclusion of MPs. Further, the mass loss from PLA membranes becomes more significant relative to PCL counterparts. Besides, such model also shows that PLA partially diminishes the release rate of TCH due to their higher interaction with $\mathrm{TCH}$ in contrast with PCL.

\section{Data Availability}

All data generated or analysed during this study are included in this manuscript.

\section{Disclosure}

A part of data in this work was previously presented by the first author at Chemeca 2014 Conference, Perth, Western Australia between September 28 and October 1, 2014.

\section{Conflicts of Interest}

The authors declare that they have no conflicts of interest and no financial interest related to this study.

\section{References}

[1] S. Heydarkhan-Hagvall, K. Schenke-Layland, A. Dhanasopon et al., "Three-dimensional electrospun ECM-based hybrid scaffolds for cardiovascular tissue engineering," Biomaterials, vol. 29, no. 19, pp. 2907-2914, 2008.

[2] K. Koenig, N. Balakrishnan, S. Hermanns, F. Langensiepen, and G. Seide, "Biobased dyes as conductive additives to reduce the diameter of polylactic acid fibers during melt electrospinning," Materials, vol. 13, no. 5, p. 1055, 2020.

[3] D. Liang, B. Hsiao, and B. Chu, "Functional electrospun nanofibrous scaffolds for biomedical applications," Advanced Drug Delivery Reviews, vol. 59, no. 14, pp. 1392-1412, 2007.

[4] S. Parham, A. Z. Kharazi, H. R. Bakhsheshi-Rad et al., "Electrospun nano-fibers for biomedical and tissue engineering applications: a comprehensive review," Materials, vol. 13, no. 9, p. 2153, 2020.

[5] E. Kenawy, F. Abdel-Hay, M. El-Newehy, and G. Wnek, "Processing of polymer nanofibers through electrospinning as drug delivery systems," Materials Chemistry and Physics, vol. 113, no. 1, pp. 296-302, 2009.

[6] H. J. Haroosh, Y. Dong, and K.-T. Lau, “Tetracycline hydrochloride (TCH)-loaded drug carrier based on PLA: PCL nanofibre mats: experimental characterisation and release kinetics modelling," Journal of Materials Science, vol. 49, no. 18, pp. 6270-6281, 2014.
[7] N. Noor Azman, S. A. Siddiqui, H. J. Haroosh et al., "Characteristics of X-ray attenuation in electrospun bismuth oxide/ polylactic acid nanofibre mats," Journal of Synchrotron Radiation, vol. 20, no. 5, pp. 741-748, 2013.

[8] Y. Dong, T. Mosaval, H. J. Haroosh, R. Umer, H. Takagi, and K. T. Lau, "The potential use of electrospun polylactic acid nanofibers as alternative reinforcements in an epoxy composite system," Journal of Polymer Science Part B: Polymer Physics, vol. 52, no. 9, pp. 618-623, 2014.

[9] Y. Dong, A. Ghataura, H. Takagi, H. J. Haroosh, A. N. Nakagaito, and K.-T. Lau, "Polylactic acid (PLA) biocomposites reinforced with coir fibres: evaluation of mechanical performance and multifunctional properties," Composites Part A: Applied Science and Manufacturing, vol. 63, pp. 76-84, 2014.

[10] H. J. Haroosh, D. S. Chaudhary, and Y. Dong, "Electrospun PLA/PCL fibers with tubular nanoclay: morphological and structural analysis," Journal of Applied Polymer Science, vol. 124, no. 5, pp. 3930-3939, 2012.

[11] B. Y. Wang, S. Z. Fu, P. Y. Ni et al., "Electrospun polylactide/ poly(ethylene glycol) hybrid fibrous scaffolds for tissue engineering," Journal of Biomedical Materials Research Part A, vol. 100A, no. 2, pp. 441-449, 2012.

[12] L. T. Lim, R. Auras, and M. Rubino, "Processing technologies for poly(lactic acid)," Progress in Polymer Science, vol. 33, no. 8, pp. 820-852, 2008.

[13] Y. Dong, D. Chaudhary, H. Haroosh, and T. Bickford, "Development and characterisation of novel electrospun polylactic acid/tubular clay nanocomposites," Journal of Materials Science, vol. 46, no. 18, pp. 6148-6153, 2011.

[14] Y. Dong, T. Bickford, H. J. Haroosh, K.-T. Lau, and H. TakagiMulti-response analysis in the material characterisation of electrospun poly(lactic acid)/halloysite nanotube composite fibres based on Taguchi design of experiments: fibre diameter, non-intercalation and nucleation effects," Applied Physics A: Materials Science \& Processing, vol. 112, no. 3, pp. 747-757, 2013.

[15] W. Chow, W. Tham, and P. Seow, "Effects of maleated-PLA compatibilizer on the properties of poly(lactic acid)/halloysite clay composites," Journal of Thermoplastic Composite Materials, vol. 26, no. 10, pp. 1349-1363, 2013.

[16] A. Touny, J. Lawrence, A. Jones, and S. Bhaduri, "Effect of electrospinning parameters on the characterization of PLA/HNT nanocomposite fibers," Journal of Materials Research, vol. 25, no. 5, pp. 857-865, 2010.

[17] X. Xu, W. Zhong, S. Zhou, A. Trajtman, and M. Alfa, "Electrospun PEG-PLA nanofibrous membrane for sustained release of hydrophilic antibiotics," Journal of Applied Polymer Science, vol. 118, no. 1, pp. 588-595, 2010.

[18] K. Lee, H. Kim, M. Khil, Y. Ra, and D. Lee, "Characterization of nano-structured poly( $\varepsilon$-caprolactone) nonwoven mats via electrospinning," Polymer, vol. 44, no. 4, pp. 12871294, 2003.

[19] H. J. Haroosh, Y. Dong, D. S. Chaudhary, G. D. Ingram, and S. Yusa, "Electrospun PLA: PCL composites embedded with unmodified and 3-aminopropyltriethoxysilane (ASP) modified halloysite nanotubes (HNT)," Applied Physics A: Materials Science \& Processing, vol. 110, no. 2, pp. 433-442, 2013.

[20] S. Unal, S. Arslan, B. K. Yilmaz et al., "Polycaprolactone/gelatin/hyaluronic acid electrospun scaffolds to mimic glioblastoma extracellular matrix," Materials, vol. 13, no. 11, p. 2661, 2020. 
[21] E. Luong-Van, L. Grøndahl, K. Chua, K. Leong, V. Nurcombe, and S. Cool, "Controlled release of heparin from poly( $\varepsilon_{-}$ -caprolactone) electrospun fibers," Biomaterials, vol. 27, no. 9, pp. 2042-2050, 2006.

[22] M. P. Arrieta, A. Leonés Gil, M. Yusef, J. M. Kenny, and L. Peponi, "Electrospinning of PCL-based blends: processing optimization for their scalable production," Materials, vol. 13, no. 17, p. 3853, 2020.

[23] Z. Huang, C. He, A. Yang et al., "Encapsulating drugs in biodegradable ultrafine fibers through co-axial electrospinning," Journal of Biomedical Materials Research Part A, vol. 77A, no. 1, pp. 169-179, 2006.

[24] Z. Meng, W. Zheng, L. Li, and Y. Zheng, "Fabrication and characterization of three-dimensional nanofiber membrance of PCL-MWCNTs by electrospinning," Materials Science and Engineering: C Materials for Biological Applications, vol. 30, no. 7, pp. 1014-1021, 2010.

[25] C. R. Lin, T. C. Tsai, M. Chung, and S. Z. Lu, "Synthesis and characterization of magnetic nanoparticles embedded in polyvinyl pyrrolidone nanofiber film by electrospinning method," Journal of Applied Physics, vol. 105, no. 7, p. 07B509, 2009.

[26] Y. K. Sung, B. W. Ahn, and T. J. Kang, "Magnetic nanofibers with core $\left(\mathrm{Fe}_{3} \mathrm{O}_{4}\right.$ nanoparticle suspension)/sheath (poly ethylene terephthalate) structure fabricated by coaxial electrospinning," Journal of Magnetism and Magnetic Materials, vol. 324, no. 6, pp. 916-922, 2012.

[27] M. Hamoudeh and H. Fessi, "Preparation, characterization and surface study of poly-epsilon caprolactone magnetic microparticles," Journal of Colloid and Interface Science, vol. 300, no. 2, pp. 584-590, 2006.

[28] M. Chowdhury and G. Stylios, "Process optimization and alignment of PVA/FeCl 3 nano composite fibres by electrospinning," Journal of Materials Science, vol. 46, no. 10, pp. 3378-3386, 2011.

[29] L. Zeng, L. An, and X. Wu, "Modeling drug-carrier interaction in the drug release from nanocarriers," Journal of Drug Delivery, vol. 2011, Article ID 370308, 15 pages, 2011.

[30] H. J. Haroosh, Y. Dong, and G. D. Ingram, "Synthesis, morphological structures, and material characterization of electrospun PLA: PCL/magnetic nanoparticle composites for drug delivery," Journal of Polymer Science Part B: Polymer Physics, vol. 51, no. 22, pp. 1607-1617, 2013.

[31] S. A. Sassman and L. S. Lee, "Sorption of three tetracyclines by several soils: assessing the role of $\mathrm{pH}$ and cation exchange," Environmental Science \& Technology, vol. 39, no. 19, pp. 7452-7459, 2005.

[32] H. J. Haroosh, Y. Dong, S. Jasim, and S. Ramakrishna, "Improvement of drug release and compatibility between hydrophilic drugs and hydrophobic nanofibrous composites," Materials, vol. 14, no. 18, p. 5344, 2021.

[33] M. Shabanian, N.-J. Kang, D.-Y. Wang, U. Wagenknecht, and G. Heinrich, "Synthesis of aromatic-aliphatic polyamide acting as adjuvant in polylactic acid (PLA)/ammonium polyphosphate (APP) system," Polymer Degradation and Stability, vol. 98, no. 5, pp. 1036-1042, 2013.
[34] R. V. Kumar, Y. Koltypin, Y. S. Cohen et al., "Preparation of amorphous magnetite nanoparticles embedded in polyvinyl alcohol using ultrasound radiation," Journal of Materials Chemistry, vol. 10, no. 5, pp. 1125-1129, 2000.

[35] D. Puppi, A. Piras, N. Detta, D. Dinucci, and F. Chiellini, "Poly(lactic-_co-_ glycolic acid) electrospun fibrous meshes for the controlled release of retinoic acid," Acta Biomaterialia, vol. 6, no. 4, pp. 1258-1268, 2010.

[36] J. H. Lee, T. G. Park, H. S. Park et al., "Thermal and mechanical characteristics of poly(L-lactic acid) nanocomposite scaffold," Biomaterials, vol. 24, no. 16, pp. 2773-2778, 2003. 